\title{
Integrated epigenetics, transcriptomics, and metabolomics to analyze the mechanisms of Benzo[a]pyrene neurotoxicity in the hippocampus
}

Jing Wang ${ }^{1 \dagger} \cdot$ Chun-Lin $\mathrm{Li}^{1 \dagger} \cdot$ Bai-Jie $\mathrm{Tu}^{1} \cdot$ Kai Yang ${ }^{2} \cdot$ Ting-Ting Mo ${ }^{1} \cdot$ Rui-Yuan Zhang $^{1} \cdot$ Shu-Qun Cheng $^{1} \cdot$ Cheng-Zhi Chen ${ }^{1} \cdot$ Xue-Jun Jiang ${ }^{1} \cdot$ Ting-Li Han $^{3,4}$ Bin Peng ${ }^{5} \cdot$ Philip N. Baker ${ }^{6} \cdot$ Yin-Yin Xia ${ }^{1,3^{*}}$

1. Department of Occupational and Environmental Hygiene, School of Public Health and Management, Research Center for Medicine and Social Development, Innovation Center for Social Risk Governance in Health, Chongqing Medical University, Chongqing, China.

2. Centers for Disease Control and Prevention of Chengdu.

3. China-Canada-New Zealand Joint Laboratory of Maternal and Fetal Medicine, Chongqing Medical University, Chongqing, China.

4. The Liggins Institute, University of Auckland, Auckland, New Zealand.

5. Department of Statistics, School of Public Health and Management, Chongqing Medical University, Chongqing, China.

6. College of Medicine, Biological Sciences and Psychology, University of Leicester, P.O. Box 138, Leicester LE1 9HN, UK.

$\uparrow$ These authors contributed equally to this work.

* Correspondence author: Yin-Yin Xia, School of Public Health and Management, Chongqing Medical University, Yuzhong District, Chongqing, China (e-mail: kendraxia@163.com)

\begin{abstract}
Benzo[a]pyrene $(\mathrm{B}[\mathrm{a}] \mathrm{P})$ is a common environmental pollutant that is neurotoxic to mammals, which can cause changes to hippocampal function and result in cognitive disorders. The mechanisms of $\mathrm{B}[\mathrm{a}] \mathrm{P}$-induced impairments are complex .To date there have been no studies on the association of epigenetic, transcriptomic and metabolomic changes with neurotoxicity after $\mathrm{B}[\mathrm{a}] \mathrm{P}$ exposure. In the present study, we investigated
\end{abstract}


the global effect of $\mathrm{B}[\mathrm{a}] \mathrm{P}$ on DNA methylation patterns, non-coding RNAs (ncRNAs) expression, coding RNAs expression, and metabolites in the rat hippocampus. Male Sprague-Dawley rats (SD rats) received daily gavage of $\mathrm{B}[\mathrm{a}] \mathrm{P}(2.0 \mathrm{mg} / \mathrm{kg}$ body weight $[\mathrm{BW}]$ ) or corn oil for seven weeks. Learning and memory ability was analysed using the Morris Water Maze (MWM) test and change to cellular ultrastructure in the hippocampus was analysed using electron microscope observation. Integrated analysis of epigenetics, transcriptomics, and metabolomics was conducted to investigate the effect of $\mathrm{B}[\mathrm{a}] \mathrm{P}$ exposure on the signaling and metabolic pathways. Our results suggest that $\mathrm{B}[\mathrm{a}] \mathrm{P}$ could lead to learning and memory deficits, likely as a result of epigenetic and transcriptomic changes that further affected the expression of CACNA1C, Tpo, etc. The changes in expression ultimately affecting LTP, tyrosine metabolism and other important metabolic pathways.

Keywords Benzo[a]pyrene; hippocampus; neurotoxicity; epigenetics; transcriptomics; metabolomics

\section{Introduction}

Benzo[a]pyrene $(\mathrm{B}[\mathrm{a}] \mathrm{P})$, a typical polycyclic aromatic hydrocarbon $(\mathrm{PAH})$, is ubiquitous in the environmental surroundings. It is produced by incomplete combustion of organic materials, such as those produced by volcanoes, forest fires, and human-made sources like industrial processing and vehicle exhaust. Consumption via contaminated food, water, and air, makes $\mathrm{B}[\mathrm{a}] \mathrm{P}$ exposure almost inevitable (Chepelev, Moffat et al. 2015). The potential point of departure (POD) for neurotoxicity in rodents, based on a standard behavioural test (Morris Water Maze [MWM]), was as low as $0.025 \mathrm{mg} \mathrm{B}[\mathrm{a}] \mathrm{P} / \mathrm{kg}$-body weight (BW)-day compared to 0.54 $\mathrm{mg} \mathrm{B}[\mathrm{a}] \mathrm{P} / \mathrm{kg}-\mathrm{BW}$-day for rodent forestomach carcinogenicity, suggesting that neurotoxic endpoints are more sensitive to $\mathrm{B}[\mathrm{a}] \mathrm{P}$ exposure than cancer endpoints (Chepelev, Moffat et al. 2015). Therefore, neurotoxicity is worth to be paid much attention. 
Because of its lipophilicity, B[a]P and its metabolites can cross the blood-brain barrier (BBB), easily reach the brain tissue and cause impairments of the central nervous system (CNS) (Chepelev, Moffat et al. 2015, Zhang, Tian et al. 2016). The "brain growth spurt" (BGS) is the the term used to refer to critical period of brain development. This period is characterized by axonal and dendritic outgrowth, establishment of neuronal connections, synaptogenesis, and proliferation of glia cells with accompanying myelinization (Eriksson 1997, Gale, Walton et al. 2003, Viberg, Mundy et al. 2008). Early postnatal exposure to B[a]P, especially in the brain rapid development period (postnatal days [PNDs] 5-11), can cause brain damage which might persist into adolescence and adulthood (Chen, Tang et al. 2012). The hippocampus is part of the brain associated with formation of learning and memory ability and emotional responses. Learning and memory disorders have been recognized as an important manifestation of $\mathrm{B}[\mathrm{a}] \mathrm{P}$-induced hippocampal injury. Recent studies reported that $\mathrm{B}[\mathrm{a}] \mathrm{P}$ could induce learning and memory impairments, which were mainly related to N-methyl-D-aspartate receptor (NMDAR), cytochrome P450, and DNA damage (Verma, Pink et al. 2012, Einaudi, Courbiere et al. 2014, Chepelev, Moffat et al. 2015, Krais, Speksnijder et al. 2016). B[a]P-induced neurotoxicity is a complex process involving multiple genes, multiple signaling pathways, and multiple regulatory mechanisms. However, current studies of the mechanisms underlying $\mathrm{B}[\mathrm{a}] \mathrm{P}$ neurotoxicity are limited. The mechanisms of $\mathrm{B}[\mathrm{a}] \mathrm{P}$-induced impairments might include alterations of DNA methylation, ncRNAs, mRNAs, proteins and metabolites, yet very few studies on aberrant epigenetic regulation patterns in DNA methylation and ncRNAs at a global scale have been reported.

The transcriptome refers to the total number of genes expressed by a specific cell in a functional state, representing the identity and expression level of each gene (Velculescu, Zhang et al. 1997). Epigenetics refers to the changes in the gene expression in the absence of DNA sequence differences and this is mainly regulated via DNA methylation, histone modification, and ncRNA interference (Qureshi and Mehler 2011). Methylation is one of the most important mechanisms of epigenetic 
regulation found in recent years (Chen and Riggs 2011), which plays an important role in inhibiting gene expression (Fuks, Burgers et al. 2000). Studies have shown that DNA methylation is related to neuronal development and synapse formation (Liu, van Groen et al. 2009, Yu, Baek et al. 2011, Morris and Monteggia 2014), but no studies to date have explored the effect on DNA methylation after $\mathrm{B}[\mathrm{a}] \mathrm{P}$ exposure. The ncRNA is a type of RNA that lacks a protein-coding sequence but has enzymatic, structural, or regulatory function. The ncRNAs can be divided into two major classes: the small ncRNAs which include microRNAs (miRNAs) and other noncoding transcripts of less than 200 nucleotides, and long noncoding RNAs (lncRNAs) which contain from 200 to more than 100000 nucleotides (Costa 2010). The miRNAs are a group of $22 \mathrm{nt}$ length endogenous ncRNAs that act as post-transcriptional regulators of protein-coding genes by binding mainly to their 3' untranslated region (UTRs), leading to mRNA degradation or inhibited translation (Cullen 2004, Wang, Niu et al. 2011). The mode of target mRNAs regulation depends on the sequence complementarity between the miRNAs and the mRNAs (Wang, Niu et al. 2011). Hundreds of miRNAs have been identified in the mammalian CNS, some of which have been shown to play a key role in the development of neurodegenerative diseases (Madathil, Nelson et al. 2011, Bhalala, Srikanth et al. 2013, Drusco, Bottoni et al. 2015). IncRNAs, one of the most abundant classes of ncRNAs, are encoded within the genome and are highly expressed in the brain (Ponjavic, Oliver et al. 2009). In contrast to miRNAs, lncRNAs have roles in diverse cellular processes and biological function. For example, lncRNAs can serve as mediators of mRNA decay, as structural scaffolds for nuclear substructures, as host genes for miRNAs, and as regulators of chromatin remodeling (Wilusz, Sunwoo et al. 2009). Although studies have shown that lncRNAs play an important role in neurological development, stress responses and plasticity, little is known about the involvement of lncRNAs in the physiology and pathology of the nervous system (Qureshi, Mattick et al. 2010). In addition, the roles of miRNAs and lncRNAs in B[a]P-induced learning and memory dysfunction are poorly understood. 
Metabolomics aims to characterize and quantify all the small molecules in a complex multicellular system to understand systematic changes through time (Nicholson and Lindon 2008). Metabolomics has previously been applied to elucidate metabolite changes in the brain of animals which were exposed to neurotoxins, and for the identification of early differential markers for neurological diseases $(\mathrm{Xu}$, Sun et al. 2015, Zaitsu, Hayashi et al. 2015, Wen, Zhang et al. 2016). In recent years, some metabolomics researchers have discovered adverse effects of $\mathrm{B}[\mathrm{a}] \mathrm{P}$ by studying Manila clam, Pinctada martensii, Hepalclc7 cells, human cells and serum (Zhang, Liu et al. 2011, Pink, Verma et al. 2014, Kalkhof, Dautel et al. 2015, Wang, Zhang et al.

2015, Ba, Huang et al. 2016, Chen, Song et al. 2016), but only a few studies have analyzed the metabolome to study the neurotoxicity of $\mathrm{B}[\mathrm{a}] \mathrm{P}$ and to identify potential biomarkers for its effects on the brain.

In this study, DNA methylation, ncRNAs, mRNAs and metabolite changes are investigated in the rat hippocampus following $\mathrm{B}[\mathrm{a}] \mathrm{P}$ treatment. The results of this study will be helpful to gain comprehensive insights into the mechanisms underlying $\mathrm{B}[\mathrm{a}] \mathrm{P}$-induced neurotoxicity. This is the first report to describe transcriptome mRNAs, methylated ncRNAs, and metabolite-wide changes in B[a]P-treated rats.

\section{Materials and methods}

\section{Materials}

Benzo[a]pyrene ( $>96 \%$ pure), the derivatization reagent methyl chloroformate (MCF), the internal standard D4-L-alanine, and pyridine were purchased from Sigma-Aldrich (St. Louis, USA). Methanol, chloroform, sodium hydroxide, and sodium bicarbonate were obtained from Merck (Darmastadt, Germany). Anhydrous sodium sulfate was purchased from Fluka (Steinheim, Germany). High-throughput sequencing reagent HiSeq Rapid SBS Kit V2 (200 cycle), HiSeq Rapid PE Cluster Kit V2, HiSeq 3000/4000 SBS Kit (300 Cycles), HiSeq 3000/4000 PE Cluster Kit, HiSeq Rapid SBS Kit V2 (50 cycle) HiSeq, Rapid SR Cluster Kit V2, and EpicentreRibo-Zero rRNA Removal Kit were purchased from Illumina (San Diego, USA). High Sensitivity D1000 Reagent and High Sensitivity D1000 ScreenTape were obtained from Agilent 
Technologies (Paro Ato, USA). NEBNext ${ }^{\circledR}$ Multiplex Small RNA Library Prep Set for Illumina $^{\circledR}$, NEBNext $^{\circledR}$ Ultra $^{\text {TM }}$ RNA Library Prep Kit for Illumina, NEBNext ${ }^{\circledR}$ Ultra $^{\text {TM }}$ DNA Library Prep Kit for Illumina and MspI were purchased from NEB (Texas, USA). Qubit ${ }^{\circledR}$ dsDNA HS Assay Kit and TRIzol reagent were purchased from Life Technologies (Carlsbad, USA). RNA ScreenTape, RNA Reagent, D1000 ScreenTape, and D1000 Reagent were obtained from Agilent Technologies (El Paso, USA). QIA quick PCR Purification kit and QIAquickMinElute Gel Extraction Kit were obtained from QIAGEN (Nordrhein-Westfalen, Germany). EZ DNA Methylation-God kit was purchased from Zymo Research (Orange, USA). All the reagents in this research were of analytical grade.

\section{Animals and Treatment}

A total of 40 four-day-old neonatal male Sprague-Dawley rats (SD rats) were obtained from Chongqing Medical University Laboratory Animal Center, China [certificate: SCXK (YU) 2007-0001]. The biological age and treatment period of the rats was selected according to the biological age of the mammalian brain development period (Spear 2007, McCallister, Li et al. 2015). Rats were housed in an environment controlled room (temperature $23 \pm 1 \square ; 12 \mathrm{~h}$ light/12 h dark cycle) with free access to food and water. After one day of acclimatization to the surroundings, the rats were randomly divided into two groups: control group $(n=20)$ and $\mathrm{B}[\mathrm{a}] \mathrm{P}-$ treated group $(n$ $=20)$.

Other than the $\mathrm{B}[\mathrm{a}] \mathrm{P}$ treatment, both groups were treated indiscriminatingly. $\mathrm{B}[\mathrm{a}] \mathrm{P}$ was dissolved in corn oil due to its lipophilicity and insolubility in water, and the daily equivalent volume of corn oil was given to the control group. The B[a]P-treated group received oral gavage of $\mathrm{B}[\mathrm{a}] \mathrm{P}$ solution $(1.0 \mathrm{~mL} / \mathrm{kg}$ body weight $)$ at a concentration of $2.0 \mathrm{mg} \mathrm{B}[\mathrm{a}] \mathrm{P} / \mathrm{kg}$, once daily, for seven consecutive weeks. This dose was selected based on the level of human environmental $\mathrm{B}[\mathrm{a}] \mathrm{P}$ exposure and is a dose that has been used in a previously published study (Chengzhi, Yan et al. 2011). Oral gavage was chosen for administration because of its resemblance with the most common form of human exposure (McCallister, Li et al. 2015). The BGS that occurs 
in neonatal rats reaches its peak around PND 10, and throughout the first 3-4 weeks of life (Eriksson 1997, Gale, Walton et al. 2003, Viberg, Mundy et al. 2008). PND 5-10 is considered the rapid brain development period (Chen, Tang et al. 2012), therefore, our treatment began at PND 5 and lasted seven weeks, to simulate the persistent exposure to $\mathrm{B}[\mathrm{a}] \mathrm{P}$ of infants and children before adulthood. Pups of both groups were weaned on PND 21 and redistributed to their corresponding cages.

The study was conducted in compliance with standard animal use practices and approved by the Ethics Committee of the Chongqing Medical University.

\section{Morris Water Maze (MWM) test}

After completing the $\mathrm{B}[\mathrm{a}] \mathrm{P}$ treatment for seven weeks (49 days), spatial learning and memory abilities of both groups ( $n=8$ in each group) were assessed by the MWM, as previously described (Chengzhi, Yan et al. 2011). A circular pool (diameter $130 \mathrm{~cm}$ ) was filled with water which was made opaque with milk powder $\left(22^{\circ} \mathrm{C} \pm 1{ }^{\circ} \mathrm{C}\right)$ and rats were trained to pinpoint a submerged platform. Rats were first trained to pinpoint a clearly marked platform (visible platform, Day 1), which was subsequently changed to a hidden platform beneath the surface of the opaque water (hidden platform, Days 2-4). There were four daily trials for both visible and hidden sessions. The rats were placed into the water facing the edge of the pool in one of four randomized locations. The beginning location was changed for each trial. A trial ended when the rats located the platform. Rats that failed to find the platform within $60 \mathrm{~s}$ were led to the platform by placing a finger in front of their swim path. The rats were taken out of the pool after they physically stayed on the platform for a minimum of $3 \mathrm{~s}$.

During visible platform training, the platform was moved to a different quadrant of the pool for each session. For the hidden platform training, the platform location was kept constant. Training during the hidden platform sessions required the rats to learn to find the location of the hidden platform based on extramaze cues. The platform was removed from the pool at Day 5. The rats were then randomly released at any one of the edges and allowed to swim for $120 \mathrm{~s}$ in order to test memory retention. The number of times that the rats crossed the target quadrant which the platform had 
previously been placed and the amount of time that the rats spent in the target quadrant were recorded. The swimming patterns of the rats were recorded by a video tracking software.

\section{Sample Preparation for Electron Microscopy}

Rats from both groups ( $n=3$ from each group) were randomly chosen and used for transmission electron microscopy, as previously described (Chengzhi, Yan et al. 2011). The rats were perfused with phosphate-buffered saline, followed by a combined fixative solution of phosphate-buffered $2.5 \%$ glutaraldehyde and $4 \%$ paraformaldehyde, after which a fixed hippocampus was acquired. Subsequently, the hippocampus was sliced into sections of approximately $1 \mathrm{~mm}^{2}$, fixed in $2.5 \%$ glutaraldehyde for $12 \mathrm{~h}$, and then fixed in $1 \%$ osmium tetroxide for $2 \mathrm{~h}$. The fixed specimen was dehydrated in a series of increasing concentrations of alcohol solutions, immersed in propylene oxide, and embedded in 618. Each section was placed on a glass slide and was stained with uranyl acetate and lead citrate, ready for observation by transmission electron microscope.

Synapses of each group were used for the average thickness of postsynaptic density (PSD) analysis via ImageJ software, as previously described (Dosemeci, Tao-Cheng et al. 2001). To calculate the average PSD thickness for each synapse, the outline of the PSD was traced and divided by length of the PSD.

\section{High-throughput sequencing and data analysis}

Hippocampus tissues from both groups ( $n=3$ from each group) were randomly selected for high-throughput sequencing, as previously described (Lu, Qin et al. 2016). Hippocampus tissues were disrupted and homogeneously mixed to make two pools representing the two groups (Zhang, Zhao et al. 2014). For each pooled sample, approximately $25 \mathrm{mg}$ and $40 \mathrm{mg}$ of tissue was used for preparation of desoxyribouncleic acid (DNA) and ribouncleic acid (RNA), respectively. The quantity and quality of total DNA and RNA were measured by ND-1000 Nanodrop (Thermo Fisher, USA) and Agilent 2200 TapeStation (Agilent, USA). Subsequently, the 
reduced representation bisulfite sequencing (RRBS) libraries, RNA-seq libraries, and miRNA-Seq libraries were constructed in this study. The quality of the libraries was assessed on the Agilent 2200 TapeStation (Agilent, USA). After cluster generation, the libraries were sequenced on Hiseq 2500 platform (Illumina, USA). High-throughput sequencing was performed by Ruibo Biotechnology Ltd. Co, Guangzhou, China.

Annotation files of mRNAs and lncRNAs were downloaded from GENCODE rn6, miRNAs annotation data was retrieved from miRBase21, and DNA annotation data was retrieved from GENCODE rn5.

\section{Whole genome methylation level and ncRNAs methylation profiles}

The DNA, lncRNA, and primary miRNA annotation files of rats were downloaded, as mentioned above. The Bismark software was used to compare the sequence acquired by RRBS with the reference genomes of these regions, to obtain methylation sites and methylation regions. Each C-base methylation site was annotated by ANNOVAR software for genomic elements. Finally, two R language packages, MetylKit and eDMR, were combined for inter-organizational differential methylation regions (DMR) analysis.

The IncRNA and miRNA promoters were defined as $10 \mathrm{~kb}$ upstream from the transcriptional start site (TSS) of each lncRNA or pri-miRNA (Xiao, Cao et al. 2017). For each sample, lncRNA or miRNA methylation profiles were contructed using the methylation levels of the promoter region of the lncRNAs or miRNAs.

\section{Genes targeted by the differentially expressed methylated ncRNAs}

Three online databases were searched to find the predicted miRNAs target genes: miRanda (http://www.microrna.org/microrna/getGeneForm.do), miRDB $\begin{array}{llll}\text { (http://www.mirdb.org/miRDB/index.html), } & \text { and }\end{array}$ (http://www.targetscan.org/vert_71/). The miRNAs target genes are the result co-predicted by the three databases. The role of lncRNAs and target genes can be grouped into two major classes: Cis and Trans. The mechanism of Cis is to search all 
the encoded genes in the $10 \mathrm{~K}$ up and down range of lncRNAs, and to overlap the genes that have significant functional expression with the lncRNA. These genes, which are near and expressed on the genome, are likely to be regulated by the lncRNA. The Trans effect is to first extract the sequence of lncRNAs and mRNAs, Blast (e $<1 \mathrm{e}-5)$ screening, and then use Rnaplex $(\mathrm{g}<-20)$ software for filtration, to identify possible target genes for lncRNA.

PANTHER pathway and Kyoto Encyclopedia of Genes and Genomes (KEGG) pathway analysis of mRNA and target genes for methylated miRNAs and IncRNAs

KOBAS (Database for Annotation, Visualization and Integrated Discovery, http://kobas.cbi.pku.edu.cn/) was used to analyze differentially expressed genes (DEG) and differentially expressed methylated ncRNAs. PANTHER pathway and KEGG pathway analysis was performed to determine the involvement of DEG and differentially expressed methylated ncRNAs in various biological pathways. PANTHER pathways and KEGG pathways with $P<0.05$ were selected.

\section{Sample preparation for gas chromatography/mass spectrometry (GC-MS) analysis}

The hippocampus (mass $=50 \mathrm{mg} \pm 0.5 \mathrm{mg}$ ) of two groups ( $n=6$ from each group) were homogenized with $1.5 \mathrm{~mL}$ of prechilled methanol/water (1: 1, V: V) and $20 \mu L$ internal standard in a $2 \mathrm{~mL}$ centrifuge tube. The samples were centrifuged (16000 g, $10 \mathrm{~min}, 4^{\circ} \mathrm{C}$ ) and the supernatant from each sample was transferred into a new $2 \mathrm{~mL}$ Eppendorf tube. Solid precipitates were homogenized with $1.6 \mathrm{~mL}$ of prechilled dichloromethane/methanol (3: $1, \mathrm{~V}: \mathrm{V})$, and centrifuged $\left(16000 \mathrm{~g}, 10 \mathrm{~min}, 4^{\circ} \mathrm{C}\right)$ to obtain the supernatant. The two supernatants were collected and dried in a vacuum concentrator. To each dried sample $200 \mu L$ sodium hydroxide was added and the sample was resuspended. The resuspension was then transferred to a silanised glass tube where $167 \mu \mathrm{L}$ methanol and $34 \mu \mathrm{L}$ pyridine was added, followed by $20 \mu \mathrm{L}$ of MCF. The sample was mixed for $30 \mathrm{~s}$, followed by another addition of $20 \mu \mathrm{L} \mathrm{MCF}$, 
which was again mixed for $30 \mathrm{~s}$. To separate the MCF derivatives $400 \mu \mathrm{L}$ chloroform and $400 \mu \mathrm{L}$ of $50 \mathrm{mM}$ sodium bicarbonate was added, and mixed for $10 \mathrm{~s}$ after each addition. After being centrifuged (1500 rpm, $10 \mathrm{~min}$ ), the aqueous layer was discarded and the samples were dehydrated with anhydrous sodium sulphate before being transferred to GC vials ready for analysis (Dai, Xia et al. 2016, Li, Wang et al. 2017).

\section{GC-MS Analysis}

GC-MS analysis was performed on an Agilent 7890A gas chromatography system coupled with an Agilent 5975C mass spectrometer (Agilent, USA). The system utilized a fused silica Zebron ZB-1701 capillary column $(30 \mathrm{~m} \times 250 \mu \mathrm{m} \times 0.15 \mu \mathrm{m})$ coated with $86 \%$ dimethylpolysiloxane and 14\% cyanopropylphenyl (Phenomenex, Torrance, CA, USA). A $1.0 u \mathrm{~L}$ aliquot of sample was injected under split-less mode with the inlet temperature being set to $290^{\circ} \mathrm{C}$. The carrier gas was instrument grade helium $(99.99 \%$, BOC), and the front inlet purge flow was set to $25 \mathrm{~mL} / \mathrm{min}$ a minute after injection. The initial temperature was kept at $45^{\circ} \mathrm{C}$ for $2 \mathrm{~min}$, raised to $180^{\circ} \mathrm{C}$ at a rate of $9^{\circ} \mathrm{C} / \mathrm{min}$, held for $5 \mathrm{~min}$; increased to $220^{\circ} \mathrm{C}$ at a rate of $40^{\circ} \mathrm{C}$, held for $5 \mathrm{~min}$; increased to $240^{\circ} \mathrm{C}$ at a rate of $40^{\circ} \mathrm{C}$, held for $11.5 \mathrm{~min}$; finally increased to $280^{\circ} \mathrm{C}$ at a rate of $40^{\circ} \mathrm{C}$, held for $2 \mathrm{~min}$. The transfer line, source, and quadrupole temperature were $250^{\circ} \mathrm{C}, 230^{\circ} \mathrm{C}$, and $150^{\circ} \mathrm{C}$, respectively. The detector was run in positive ionization mode with electron energy of $70 \mathrm{eV}$ and was turned on $5.5 \mathrm{~min}$ into the run. The mass spectrometry data was acquired in scan mode with the range from $38 \mathrm{~m} / \mathrm{z}$ to $550 \mathrm{~m} / \mathrm{z}$ with scan time $0.1 \mathrm{~s}$, scan speed $1562 \mathrm{u} / \mathrm{s}$, and 100 ion counts as the detection threshold. A solvent blank was run after every six samples to check for instrument carryover (Li, Wang et al. 2017).

\section{Metabolomics Date Analysis}

Raw data obtained via GC-MS was converted into a common data format (CDF) by the Automated Mass Deconvolution and Identification System (AMDIS-Http://www.amdis.net/). Metabolites in the hippocampus were identified using an in-house mass spectral library and the NIST 0.5 library. The results included 
sample information, retention time, and peak intensities. Compounds with a match factor $>70 \%$ to one of the libraries were included in the analysis. Relative quantification of peak intensities was used to determine levels of the metabolites. The data was normalized using the internal standard and brain tissue mass. Partial least squares discriminate analysis (PLS-DA) was performed using SIMCA-P 11.5 (Umetrics, Umea, Sweden). Potential biomarkers were selected based on the variable important in the projection (VIP) values (VIP $>1)$ and statistical significance $(P<$ 0.05 ) following a Student's $t$-test, which was conducted in SPSS 22.0 (SPSS, Inc, Chicago, IL) (Li, Wang et al. 2017).

\section{Metabolic pathway analysis and visualization}

Enrichment analysis and pathway topology analysis were performed in metaboanalyst (http://www.metaboanalyst.ca/) to identify specific metabolic pathways that were associated with $\mathrm{B}[\mathrm{a}] \mathrm{P}$ toxicity. Based on the pathway information from KEGG (http://www.kegg.jp/kegg/pathway.html), metabolic and enzyme gene networks were generated by Metscape (a plug-in of Cytoscape).

\section{Statistical analysis}

Data is presented as mean \pm standard deviation $(S D)$. Statistical analysis was performed using SPSS 22.0 (SPSS, Inc, Chicago, IL). All data from the MWM was analyzed using an unpaired Student's $t$-test, with the exception of a one-way ANOVA followed by an SNK test, which was used for the comparisons among the four training days. $P<0.05$ was considered to be statistically significant. Enrichment analysis was performed using a Fisher's exact test. Genes with $P<0.05$ were considered DEG, and PANTHER pathways and KEGG pathways with $P<0.05$ were considered enriched.

\section{Results}

\section{Body weight (BW)}

The weights of rats are showed (Table 1). The $\mathrm{B}[\mathrm{a}] \mathrm{P}$-treated group weighed 
significantly less than the control group $(P<0.05)$.

\section{MWM}

Data acquired from the place navigation task for control rats and $\mathrm{B}[\mathrm{a}] \mathrm{P}-\mathrm{treated}$ rats, showed no significant difference of the swimming speed on different days (Fig. 1A). $\mathrm{B}[\mathrm{a}] \mathrm{P}$-treated rats showed a significant increase in the escape latency compared to control rats on each trial day (Fig. 1B) $(P<0.05)$. Compared with the control rats, the number of platform crossings (Fig. 1C, E) and the time in the target area (Fig. 1D, E) was significantly decreased in the $\mathrm{B}[\mathrm{a}] \mathrm{P}$-treated rats $(P<0.05)$. Results of the MWM showed that $\mathrm{B}[\mathrm{a}] \mathrm{P}$ could cause spatial learning disabilities.

\section{Ultrastructural feature in the hippocampus}

The transmission electron microscope was used to capture any ultrastructural alterations of the hippocampus after the seven weeks of B[a]P exposure. Swelled mitochondria and stratified and collapsed myelin sheaths were found in $\mathrm{B}[\mathrm{a}] \mathrm{P}-$ treated rats compared with the control group. In addition, observably thickened PSD were also found in $\mathrm{B}[\mathrm{a}] \mathrm{P}$-treated rats $(P=0.039)$ (Fig. 2).

\section{Expression profiles of miRNAs, mRNAs and IncRNAs in hippocampus of SD rats}

In total, 765 miRNAs, 50,615 mRNAs, and 16,113 lncRNAs were detected and the whole expression profile was presented (Fig. 3A, B, C). Compared with the control group, 45 miRNAs, 1566 mRNAs, and 819 lncRNAs were differentially expressed $(\log 2($ Fold change $)>1.0, P<0.05)$ in the $\mathrm{B}[\mathrm{a}] \mathrm{P}$-treated group, of which 14 miRNAs, 586 mRNAs, and 508 lncRNAs were up-regulated, and 31 miRNAs, 980 mRNAs, and 311 lncRNAs were down-regulated. In addition, 925 DEGs were obtained based on the differentially expressed mRNAs, and $\mathrm{B}[\mathrm{a}] \mathrm{P}$-treated rats showed an up-regulated expression of CaMKII $\gamma($ Fold change $=2.43)$.

\section{The whole genome methylation level in the hippocampus of SD rats}

There were 1,974,648 C base methylation sites in the control group, and 2,081,484 in 
the $\mathrm{B}[\mathrm{a}] \mathrm{P}$-treated group. Whereas, the $\mathrm{B}[\mathrm{a}] \mathrm{P}$-treated group had less methylation sites than the control group at the $\mathrm{CpG}$ island (CGI) and promoter regions (Fig. 4A). Furthermore, it was found via ANNOVAR software that intergenic was the most prone to methylation, followed by intronic, exonic, upstream, UTR5, downstream, UTR3, ncRNA, and splicing (Fig. 4B). A total of 137 DMRs $(P<0.05)$ were obtained using $\mathrm{R}$ language analysis. The distribution of DMR and differential methylation sites (DMC) on the chromosome are shown in Figure 4C. These DMRs consisted of 166 differential methylation genes (DMG), with seven miRNAs and 86 lncRNAs promoter regions being methylated.

\section{Genes targeted by differentially expressed methylated ncRNAs}

In comparison to the control group, six miRNAs and two lncRNAs were differentially expressed $(\log 2$ (Fold change) $>1.0, P<0.05)$ among these promoter methylated miRNAs and lncRNAs in the B[a]P-treated group, including mir-293, mir-483, mir-298, mir-3553, mir-191, mir-133b, LOC100910802, and LOC100911370. 320 target genes were acquired from the miRNAs target gene prediction. Only one target gene was obtained from the lncRNAs target gene prediction.

\section{Pathway analysis of DEG and differentially expressed methylated ncRNAs predicted target genes}

KEGG and PANTHER pathway annotation was applied to DEG and differentially expressed methylated ncRNAs predicted target genes. 54 KEGG pathways and four PANTHER pathways were significantly enriched $(P<0.05)$ in the DEG analysis, most of which were linked to learning and memory, including the "Alzheimer disease-presenilin pathway" (P00004), and "Tyrosine metabolism" pathways (rno00350). KEGG and PANTHER pathways that related to learning and memory were listed in Table 2. Similarly, 26 KEGG pathways and 17 PANTHER pathways were significantly enriched $(P<0.05)$ in the analysis of differentially expressed methylated ncRNAs predicted target genes. KEGG and PANTHER pathways that related to learning and memory were listed in Table 3. 


\section{Analysis of Brain Metabolomics}

The $\mathrm{B}[\mathrm{a}] \mathrm{P}-$ treated group could be distinguished from the control group in the PLS-DA score plot, which suggested that treatment with $\mathrm{B}[\mathrm{a}] \mathrm{P}$ induces distinct changes in the brain metabolites (Fig. 5).

GC-MS metabolomic profiling of the hippocampus found a total of 120 compounds in both groups, which were used in subsequent multivariate analysis. Results of the PLS-DA showed an obvious distinction between the two groups $(\mathrm{R} 2 \mathrm{X}=0.743, \mathrm{R} 2 \mathrm{Y}=$ 0.993, Q2 = 0.596). Based on PLS-DA (VIP $>1)$ and $t$-test $(P<0.05), 21$ significant metabolites were identified (Table 4) and were displayed in a heat map (Fig. 6) to show the difference in brain metabolite levels between the two groups. Pathway analysis was performed using Metaboanalyst (http://www.metaboanalyst.ca/), which suggested that $\mathrm{B}[\mathrm{a}] \mathrm{P}$ significantly impacted a number of metabolic pathways (Table 5, Fig. 7). Enzyme gene compounds reaction network diagram of the significant metabolites was constructed using Metscape. The genes involved in the analysis of enzymes gene compounds reaction of significant metabolites were compared with the genes derived from high-throughput sequencing. The genes (Fold change $>1, P<$ 0.05 ) that were associated with enzymes gene compounds reaction of significant metabolites are shown (Fig. 8, Table 6).

\section{Discussion}

$\mathrm{B}[\mathrm{a}] \mathrm{P}$ is potential neurotoxin, and previous studies have observed its neurotoxicity in hippocampus slices, neurosecretory PC12 cells, and primary cortical and striatal neurons (McCallister, Maguire et al. 2008, Slotkin and Seidler 2009, Cheng, Xia et al. 2013). Furthermore, learning and memory deficits have also been observed in animal studies after B[a]P exposure (Qiu, Cheng et al. 2011, Xia, Cheng et al. 2011, Cheng, Xia et al. 2013). In this study, high-throughput sequencing was used to assess hippocampal DNA methylation, ncRNA expression, and gene expression. The DNA methylation level, ncRNAs profile, and gene expression profile were significantly 
altered in SD rats following $\mathrm{B}[\mathrm{a}] \mathrm{P}$ exposure. By a series of bioinformatics analysis, DEG and methylated ncRNAs target genes were shown to be significantly correlated with learning and memory deficits. These RNAs could affect not only the expression of learning and memory-related proteins that directly influence learning and memory, but also the expression of enzymes which participate in the metabolic pathways that indirectly influence study memory. Learning and memory deficits caused by B[a]P neurotoxicity involved a complex biological process that involved changes in transcriptomic, epigenetic, and metabolomic profiles of the hippocampus. And coding RNAs and ncRNAs work together, which led to pathway disorders in neurotoxic induced by $\mathrm{B}[\mathrm{a}] \mathrm{P}$.

The hippocampus is a key brain structure which plays a pivotal role in learning and memory (Maciel, Biasibetti et al. 2014, He, Ji et al. 2016). Fetuses and children are more susceptible than adults to the adverse effects of environment pollutants. Studies have observed neurotoxic effects and behavioral changes when the developing brain has been exposed to repeated oral doses of toxicants (Tchekalarova, Kubova et al. 2005, Karpova, Lindholm et al. 2009, Gassowska, Baranowska-Bosiacka et al. 2016). Nerve injury was found to be irreversible, therefore, early postnatal exposure to $\mathrm{B}[\mathrm{a}] \mathrm{P}$ could cause neurodevelopmental abnormalities and influence adolescenct and adulthood neurobehavior (Bouayed, Desor et al. 2009, Chen, Tang et al. 2012). In this study, after the rats were administered $\mathrm{B}[\mathrm{a}] \mathrm{P}$ orally at a dose of $2.0 \mathrm{mg} / \mathrm{kg} \mathrm{BW}$, they experienced significant weight loss which was consistent with previous studies (Chengzhi, Yan et al. 2011). MWM is a model to detect long-term memory in animal space learning (Chen, Zhao et al. 1999). MWM performance was significantly different between the $\mathrm{B}[\mathrm{a}] \mathrm{P}$-treated group and control group, suggesting spatial learning and memory deficits in the $\mathrm{B}[\mathrm{a}] \mathrm{P}$-treated group. Results of the present study were consistent with previous researches that $\mathrm{B}[\mathrm{a}] \mathrm{P}$ had an adverse effect in neurobehavioral function. In addition, electron micrographs also showed swelled mitochondria, stratified and collapsed myelin sheaths, and thickened PSD in the hippocampal neurons following $\mathrm{B}[\mathrm{a}] \mathrm{P}$ exposure. Mitochondrial swelling is an early manifestation of mitochondrial damage due to inflammation, and the disappearance of 
mitochondrial cristae with the development of inflammation are characteristics of apoptosis (Zhang, $\mathrm{Xu}$ et al. 2013). It has previously been reported that ultrastructural changes such as mitochondrial swelling occurred in the hippocampus of epileptic macaque, and exposure to organic dioxins could lead to inflammatory reactions such as mitochondrial swelling, and degenerative lesions such as demyelination in the hippocampus. (Chen, Liu et al. 2013, Rosinczuk, Dymarek et al. 2015). Additionally, a previous study has linked $\mathrm{B}[\mathrm{a}] \mathrm{P}$ exposure to oxidative stress in the hippocampus (Yang, Chen et al. 2017). Excessive oxygen reactive species (ROS) in the brain has been found to cause neuronal oxidative stress, and to induce and enhance neurobehavioral impairment, mainly through neuroinflammation and neuronal signal alteration (Popa-Wagner, Mitran et al. 2013). Glutathione (GSH) is an important component in the intracellular antioxidant system and plays an essential role in cell defense against ROS, because it can balance the production of ROS that is generated as part of normal cellular metabolism in the brain (Li, Wang et al. 2017). In this study, abnormal glutathione metabolism was found in the hippocampus, indicating dysregulation of the antioxidant system. In analysis of both differentially expressed methylated ncRNA predicted target genes pathways and abnormal metabolite pathways, "Glutathione metabolism" (Table 3, 5) was found simultaneously. Hippocampal ultrastructural changes induced by $\mathrm{B}[\mathrm{a}] \mathrm{P}$ exposure may be attributed to differentially expressed methylated ncRNAs that abnormally regulate the expression of anti-oxidative stress pathway-related genes, eventually resulting in oxidative stress-induced hippocampal injury.

The PSD consists of many different specialized proteins (eg, NMDAR and $\mathrm{Ca}^{2+}$ /calmodulin kinase II [CaMKII]) apposed to the postsynaptic membrane for scaffolding and signaling at the synapse (Dosemeci, Tao-Cheng et al. 2001, Sheng and Hoogenraad 2007). A preliminary mode of action (MOA) that aryl hydrocarbon receptor (AHR)-dependent modulation of the transcription of NMDAR subunits and NMDAR-mediated loss of neuronal activity and decreased Long-term potentiation (LTP) explained B[a]P-induced neurotoxicity in rodents (Chepelev, Moffat et al. 2015). LTP of the hippocampus is a molecular mechanism for information storage and 
memory, and its induction must involve the NMDAR and CaMKII (Shonesy, Jalan-Sakrikar et al. 2014). The NMDAR subunits NR1, NR2A, and NR2B in PSD are binding sites for CaMKII, and thickening of PSDs can be accounted for by an accumulation of CaMKII on their cytoplasmic faces (Shen and Meyer 1999, Dosemeci, Tao-Cheng et al. 2001). Previous research has pointed out that $\mathrm{B}[\mathrm{a}] \mathrm{P}$ exposure can downregulate NMDAR, which is critical for synaptic plasticity (Cheng, Xia et al. 2013). In this study, B[a]P-treated rats showed the up-regulated expression of CaMKII $\gamma$. Four genes encode isoforms of CaMKII $(\alpha, \beta, \gamma, \delta)$, with CaMKII $\alpha$, CaMKII $\beta$, and CaMKII $\gamma$ being related to cognitive impairment (Coyle 2009, Bachstetter, Webster et al. 2014). It was speculated that $\mathrm{B}[\mathrm{a}] \mathrm{P}$ exposure influenced the normal binding of CaMKII $\gamma$ and NMDAR, resulting in deposition of CaMKII $\gamma$ on the postsynaptic membrane, leading to PSD thickening, and ultimately affecting the formation of LTP and learning and memory. Therefore, further studies are warranted to confirm the relationship between CaMKII $\gamma$ and cognitive impairment induced by $\mathrm{B}[\mathrm{a}] \mathrm{P}$ exposure.

Obvious changes in pathological lesions found in the study were consistent with changes in learning and memory function, Moreover, ultrastructure changes of to the hippocampal neurons were the structural basis of hippocampal dysfunction.

In recent years, the study of the molecular mechanisms of learning and memory has made great progress, demonstrating that learning and memory are closely related to various physiological processes, such as the release of transmitters, the calcium/kinase phosphatase system, synaptic plasticity, gene transcription and translation, DNA methylation, and so forth (Bradshaw, Kubota et al. 2003, Xia, Cheng et al. 2011, Cheng, Xia et al. 2013, Moore, Le et al. 2013, Pooters, Van der Jeugd et al. 2015). In this study, it was found that there were significantly more DNA methylation sites in the $\mathrm{B}[\mathrm{a}] \mathrm{P}$-treated rat hippocampus when compared with the control group. $\mathrm{B}[\mathrm{a}] \mathrm{P}$ exposure induced abnormal methylation patterns that might influence the differentiation and maturation of the mammalian $\mathrm{CNS}$, and regulate long-term synaptic plasticity by changing gene expression (Moore, Le et al. 2013, Senut, Sen et al. 2014). The present study also found that compared with the control group, 
expression of mRNAs and miRNAs in the B[a]P-treated group was generally down-regulated, while expression of IncRNAs was up-regulated. Both miRNAs and lncRNAs affected the synaptic plasticity by regulating the translation of proteins or proteases that were related to learning and memory (Siegel, Obernosterer et al. 2009, Gao, Wang et al. 2010, Earls, Westmoreland et al. 2014).

According to the gene biological functional analysis, $\mathrm{B}[\mathrm{a}] \mathrm{P}$-induced impairments in learning and memory were associated with signal transduction and metabolism. The key pathways included eleven "signaling pathways", five "metabolic pathways" and other learning and memory-related pathways, such as "Cell adhesion molecules (CAMs)", "Neuroactive ligand-receptor interaction", "Cytokine-cytokine receptor interaction", and "ECM-receptor interaction" (Table 2). CAMs played an important role in axonal development, synaptic formation, and learning cognition (Jurgenson, Aonurm-Helm et al. 2010, Seyedabadi, Fakhfouri et al. 2014, Thalhammer and Cingolani 2014). Neuroactive ligand-receptor interaction was necessary in signal transduction (Cheng, Xia et al. 2013), as well as cytokine-cytokine receptor interaction and ECM-receptor interaction. Therefore, the neurotoxic lesions caused by $\mathrm{B}[\mathrm{a}] \mathrm{P}$ exposure are partly attributed to disruptions in signal transduction. It was also found that aberrant methylation of ncRNAs could perturb transduction and metabolism. Eighteen "signaling pathways", two "metabolic pathways", "Cell adhesion molecules (CAMs)", "Apoptosis-multiple species", and "Long-term potentiation" were obtained in differentially expressed methylated ncRNAs predicted target genes biological functional analysis (Table 3 ), indicating that $\mathrm{B}[\mathrm{a}] \mathrm{P}$ mediated the epigenetic expression pattern (Fig. 9). Consistent with previous studies, B[a]P exposure impaired LTP in the hippocampus (Qiu, Cheng et al. 2011) and induced neuronal apoptosis which was related to learning and memory dysfunction in rats $(\mathrm{Li}$, Peng et al. 2012). As a consequence of epigenetic modification, protein expression changes occurred due to transcriptome alterations, which might serve as one of the probable factors leading to learning and memory dysfunction (Mattick and Makunin 2006, Amaral and Mattick 2008, Chengzhi, Yan et al. 2011). 
In brief, epigenetic changes appear to be a reasonable mechanism linking $\mathrm{B}[\mathrm{a}] \mathrm{P}$ exposure to learning and memory impairment.

Metabolomic information, the ultimate result of biological function, can directly indicate abnormal physiological status as it is "downstream" from those initial changes that occur at the level of genome, transcriptome, and proteome (Huang, Luo et al. 2016). After bioinformatic analysis, metabolites in the hippocampus were investigated to further understand how $\mathrm{B}[\mathrm{a}] \mathrm{P}$ exposure could lead to learning and memory deficits. Pathway analysis of the 21 significant metabolites revealed the two pathways of "Glycolysis or Gluconeogenesis" and "Tyrosine metabolism" among the 15 metabolic pathways, to be consistent with the DEG pathway and methylated ncRNAs target gene. It was shown that these two pathways were mainly affected by transcriptome changes.

Glucose homeostasis is a crucial physiological function and any abnormalities in glycolysis or gluconeogenesis can directly affect the energy supply of cells and have dramatic consequences for brain cells (Ram'ırez, Vargas et al. 2012). Hippocampus pyramidal neurons have the highest energy requirements in the brain, when their energy requirements are insufficient, the risk of cellular dysfunction and injury, and neurodegenerative disease can increase (LaMANNA and ttARIK 1985, Kapogiannis and Mattson 2011, Bauernfeind and Babbitt 2014, Liu, $\mathrm{Gu}$ et al. 2015). Neurotransmitters are important for brain development and the function of the nervous system and cognition (Seyedabadi, Fakhfouri et al. 2014). Tyrosine participates in the synthesis of biogenic amine neurotransmitters such as dopamine (DA), norepinephrine (NE) and epinephrine (A), which are essential for learning, memory, and cognition (Welch, Meyer et al. 1975). The dysregulation of tyrosine metabolism induced by $\mathrm{B}[\mathrm{a}] \mathrm{P}$ exposure might lead to a disturbance in neurotransmitter synthesis, and disruption of neurotransmitter homeostasis in the CNS.

KEGG pathway analysis revealed that differentially expressed protease genes were involved in "Tyrosine metabolism", including Tpo, RGD1308564, Aox2, Aox3, 
ALDH3A1, and Adh4 (Supplementary table 1). PANTHER pathway analysis indicated that methylated ncRNAs target genes participated in "Glycolysis", including Hk2 and Eno2 (Supplementary table 2). The abnormal expression of protease genes could be associated with the abnormal metabolism of tyrosine and glycolysis in the hippocampus, which ultimately affected the energy supply to the hippocampus and the synthesis of neurotransmitters. Taken together, it has been speculated that epigenetic changes after $\mathrm{B}[\mathrm{a}] \mathrm{P}$ exposure might further affect protease enzyme gene expression, thus altering enzyme expression, resulting in metabolite changes, which was confirmed by the results of enzyme-gene complex reaction analysis (Fig. 8, Table 6). Synthesis of glycine was regulated by Dpep2 and Pipox, while Tpo and Cpa2 were involved in the synthesis of phenylalanine and leucine, respectively. Glycine and phenylalanine are involved in the synthesis of neurotransmitters and leucine could participate in pathways related to brain energy supply, both of which have been proposed to be closely related to brain dysfunction (Tabatabaie, Klomp et al. 2010, Dai, Xia et al. 2016, Zhang, Wang et al. 2016). Therefore, these four significant metabolite-related enzymes, Dpep2, Pipox, Tpo and Cpa2 should be the focus of future research.

In conclusion, the present study found that $\mathrm{B}[\mathrm{a}] \mathrm{P}$ exposure in rats not only influenced the levels of DNA methylation and expression profiles of ncRNAs and mRNAs, but also resulted in changes to the hippocampal metabolites. This work is the first to demonstrate the potential neurotoxicity of $\mathrm{B}[\mathrm{a}] \mathrm{P}$ from an epigenetics, transcriptomics and metabolomics perspective. These results suggest that $\mathrm{B}[\mathrm{a}] \mathrm{P}$ exposure could lead to learning and memory deficits, likely as a result of epigenetic and transcriptomic changes that further affected the expression of CACNA1C, Tpo, etc. The changes in expression ultimately affecting LTP, tyrosine metabolism, and other important metabolic pathways.

\section{Availability of supporting data}

The data supporting the results of this article are available in the GEO repository [GEO: GSE109973]. 


\section{Funding}

This study was supported by the National Natural Science Foundation of China ( No. 81502777, 81372957, 81602820, 81703187 ), the Natural Science Foundation Project of CQ CSTC ( 2015jcyjA10032, cstc2016jcyjA0223, cstc2016jcyjA0435 ), the Science-Technology Research Program of the CQ Municipal Commission of Education ( KJ1600204 ), the China State Scholarship Fund, and the 111 Project.

\section{References}

Amaral, P. P. and J. S. Mattick (2008). "Noncoding RNA in development." Mamm Genome 19(7-8): 454-492.

Ba, Q., C. Huang, Y. Fu, J. Li, J. Li, R. Chu, X. Jia and H. Wang (2016). "Cumulative metabolic effects of low-dose benzo(a)pyrene exposure on human cells." Toxicology Research 5(1): 107-115.

Bachstetter, A. D., S. J. Webster, T. Tu, D. S. Goulding, J. Haiech, D. M. Watterson and L. J. Van Eldik (2014). "Generation and behavior characterization of CaMKIlbeta knockout mice." PLoS One 9(8): e105191.

Bauernfeind, A. L. and C. C. Babbitt (2014). "The appropriation of glucose through primate neurodevelopment." J Hum Evol 77: 132-140.

Bhalala, O. G., M. Srikanth and J. A. Kessler (2013). "The emerging roles of microRNAs in CNS injuries." Nat Rev Neurol 9(6): 328-339.

Bouayed, J., F. Desor, H. Rammal, A. K. Kiemer, E. Tybl, H. Schroeder, G. Rychen and R. Soulimani (2009). "Effects of lactational exposure to benzo[alpha]pyrene (B[alpha]P) on postnatal neurodevelopment, neuronal receptor gene expression and behaviour in mice." Toxicology 259(3): 97-106.

Bradshaw, J. M., Y. Kubota, T. Meyer and H. Schulman (2003). "An ultrasensitive $\mathrm{Ca} 2+/$ calmodulin-dependent protein kinase II-protein phosphatase 1 switch facilitates specificity in postsynaptic calcium signaling." Proc Natl Acad Sci U S A 100(18): 10512-10517.

Chen, C., Y. Tang, X. Jiang, Y. Qi, S. Cheng, C. Qiu, B. Peng and B. Tu (2012). "Early postnatal benzo(a)pyrene exposure in Sprague-Dawley rats causes persistent neurobehavioral impairments that emerge postnatally and continue into adolescence and adulthood." Toxicol Sci 125(1): 248-261.

Chen, H., Q. Song, X. Diao and H. Zhou (2016). "Proteomic and metabolomic analysis on the toxicological effects of Benzo[a]pyrene in pearl oyster Pinctada martensii." Aquat Toxicol 175: 81-89.

Chen, N., C. Liu, N. Yan, W. Hu, J. G. Zhang, Y. Ge and F. G. Meng (2013). "A macaque model of mesial temporal lobe epilepsy induced by unilateral intrahippocampal injection of kainic Acid." PLoS One 8(8): e72336.

Chen, Z., Q. Zhao, Y. Sugimoto, Y. Fujii and C. Kamei (1999). "Effects of histamine on MK-801-induced memory deficits in radial maze performance in rats." Brain Res 839(1): 186-189.

Chen, Z. X. and A. D. Riggs (2011). "DNA methylation and demethylation in mammals." J Biol Chem 286(21): 18347-18353.

Cheng, S. Q., Y. Y. Xia, J. L. He, X. Q. Liu, X. M. Chen, Y. B. Ding, Y. X. Wang, B. Peng and B. J. Tu (2013). 
"Neurotoxic effect of subacute benzo(a)pyrene exposure on gene and protein expression in Sprague-Dawley rats." Environ Toxicol Pharmacol 36(2): 648-658.

Chengzhi, C., T. Yan, C. Shuqun, J. Xuejun, Q. Youbin, X. Yinyin, T. Qian and T. Baijie (2011). "New candidate proteins for Benzo(a)pyrene-induced spatial learning and memoru deficits." J. Toxicol. Sci 36(2): 163-171.

Chepelev, N. L., I. D. Moffat, W. J. Bowers and C. L. Yauk (2015). "Neurotoxicity may be an overlooked consequence of benzo[a]pyrene exposure that is relevant to human health risk assessment." Mutat Res Rev Mutat Res 764: 64-89.

Costa, F. F. (2010). "Non-coding RNAs: Meet thy masters." Bioessays 32(7): 599-608.

Coyle, J. T. (2009). "MicroRNAs suggest a new mechanism for altered brain gene expression in schizophrenia." Proc Natl Acad Sci U S A 106(9): 2975-2976.

Cullen, B. R. (2004). "Transcription and processing of human microRNA precursors." Mol Cell 16(6): 861-865.

Dai, H., Y. Y. Xia, T. H. Ting-Li Han, N. B. P. N. Philip, X. Tang, R. Y. Zhang, H. Du, T. J. Cai and S. Q. Cheng (2016). "Effect of chronic arsenic exposure on mouse brain tissue and serum metabolomics." Nan Fang Yi Ke Da Xue Xue Bao 36(9): 1192-1197.

Dosemeci, A., J. H. Tao-Cheng, L. Vinade, C. A. Winters, L. Pozzo-Miller and T. S. Reese (2001). "Glutamate-induced transient modification of the postsynaptic density." Proc Natl Acad Sci U S A 98(18): 10428-10432.

Drusco, A., A. Bottoni, A. Laganà, M. Acunzo, M. Fassan, L. Cascione, A. Antenucci, P. Kumchala, C. Vicentini, M. P. Gardiman, H. Alder, M. A. Carosi, M. Ammirati, S. Gherardi, M. Luscrì, C. Carapella, NicolaZanesi and C. M. Croce (2015). "A differentially expressed set of microRNAs in cerebro-spinal fluid (CSF) can diagnose CNS malignancies." Oncotarget 6(25): 20829-20839.

Earls, L. R., J. J. Westmoreland and S. S. Zakharenko (2014). "Non-coding RNA regulation of synaptic plasticity and memory: implications for aging." Ageing Res Rev 17: 34-42.

Einaudi, L., B. Courbiere, V. Tassistro, C. Prevot, I. Sari-Minodier, T. Orsiere and J. Perrin (2014). "In vivo exposure to benzo(a)pyrene induces significant DNA damage in mouse oocytes and cumulus cells." Hum Reprod 29(3): 548-554.

Eriksson, P. (1997). "Developmental neurotoxicity of environmental agents in the neonate." Neurotoxicology 18(3): 719-726.

Fuks, F., W. A. Burgers, A. Brehm, L. Hughes-Davies and T. Kouzarides (2000). "DNA methyltransferase Dnmt1 associates with histone deacetylase activity." Nat Genet 24(1): 88-91.

Gale, C. R., S. Walton and C. N. Martyn (2003). "Foetal and postnatal head growth and risk of cognitive decline in old age." Brain 126(Pt 10): 2273-2278.

Gao, J., W. Y. Wang, Y. W. Mao, J. Graff, J. S. Guan, L. Pan, G. Mak, D. Kim, S. C. Su and L. H. Tsai (2010). "A novel pathway regulates memory and plasticity via SIRT1 and miR-134." Nature 466(7310): 1105-1109.

Gassowska, M., I. Baranowska-Bosiacka, J. Moczydlowska, M. Frontczak-Baniewicz, M. Gewartowska, L. Struzynska, I. Gutowska, D. Chlubek and A. Adamczyk (2016). "Perinatal exposure to lead (Pb) induces ultrastructural and molecular alterations in synapses of rat offspring." Toxicology.

He, J., X. Ji, Y. Li, X. Xue, G. Feng, H. Zhang, H. Wang and M. Gao (2016). "Subchronic exposure of benzo(a)pyrene interferes with the expression of Bcl-2, Ki-67, C-myc and p53, Bax, Caspase-3 in sub-regions of cerebral cortex and hippocampus." Exp Toxicol Pathol 68(2-3): 149-156.

Huang, Q., L. Luo, A. Alamdar, J. Zhang, L. Liu, M. Tian, S. A. M. A. S. Eqani and H. Shen (2016). 
"Integrated proteomics and metabolomics analysis of rat testis: Mechanism of arsenic-induced male reproductive toxicity." Scientific Reports 6(1).

Jurgenson, M., A. Aonurm-Helm and A. Zharkovsky (2010). "Behavioral profile of mice with impaired cognition in the elevated plus-maze due to a deficiency in neural cell adhesion molecule." Pharmacol Biochem Behav 96(4): 461-468.

Kalkhof, S., F. Dautel, S. Loguercio, S. Baumann, S. Trump, H. Jungnickel, W. Otto, S. Rudzok, S. Potratz, A. Luch, I. Lehmann, A. Beyer and M. von Bergen (2015). "Pathway and time-resolved benzo[a]pyrene toxicity on Hepa1c1c7 cells at toxic and subtoxic exposure." J Proteome Res 14(1): 164-182.

Kapogiannis, D. and M. P. Mattson (2011). "Disrupted energy metabolism and neuronal circuit dysfunction in cognitive impairment and Alzheimer's disease." The Lancet Neurology 10(2): 187-198.

Karpova, N. N., J. Lindholm, P. Pruunsild, T. Timmusk and E. Castren (2009). "Long-lasting behavioural and molecular alterations induced by early postnatal fluoxetine exposure are restored by chronic fluoxetine treatment in adult mice." Eur Neuropsychopharmacol 19(2): 97-108.

Krais, A. M., E. N. Speksnijder, J. P. Melis, R. Indra, M. Moserova, R. W. Godschalk, F. J. van Schooten, A. Seidel, K. Kopka, H. H. Schmeiser, M. Stiborova, D. H. Phillips, M. Luijten and V. M. Arlt (2016). "The impact of p53 on DNA damage and metabolic activation of the environmental carcinogen benzo[a]pyrene: effects in Trp53(+/+), Trp53(+/-) and Trp53(-/-) mice." Arch Toxicol 90(4): 839-851.

LaMANNA, J. C. and S. I. ttARIK (1985). "Regional comparisons of brain glucose influx." brain Research 326(1985): 299-305.

Li, C., J. Wang, Q. Su, K. Yang, C. Chen, X. Jiang, T. Han, S. Cheng, T. Mo, R. Zhang, B. Peng, Y. Guo, P. N. Baker, B. Tu and Y. Xia (2017). "Postnatal Subacute Benzo(a)Pyrene Exposure Caused Neurobehavioral Impairment and Metabolomic Changes of Cerebellum in the Early Adulthood Period of Sprague-Dawley Rats." Neurotox Res.

Li, M., J. Peng, Y. Song, H. Liang, Y. Mei and Y. Fang (2012). "Electro-acupuncture combined with transcranial magnetic stimulation improves learning and memory function of rats with cerebral infarction by inhibiting neuron cell apoptosis." J Huazhong Univ Sci Technolog Med Sci 32(5): 746-749. Liu, B., Y. Gu, H. Xiao, X. Lei, W. Liang and J. Zhang (2015). "Altered metabolomic profiles may be associated with sevoflurane-induced neurotoxicity in neonatal rats." Neurochem Res 40(4): 788-799.

Liu, L., T. van Groen, I. Kadish and T. O. Tollefsbol (2009). "DNA methylation impacts on learning and memory in aging." Neurobiol Aging 30(4): 549-560.

Lu, Y., B. Qin, H. Hu, J. Zhang, Y. Wang, Q. Wang and S. Wang (2016). "Integrative microRNA-gene expression network analysis in genetic hypercalciuric stone-forming rat kidney." PeerJ 4: e1884.

Maciel, E. S., R. Biasibetti, A. P. Costa, P. Lunardi, R. V. Schunck, G. C. Becker, M. D. Arbo, E. Dallegrave, C. A. Goncalves, P. H. Saldiva, S. C. Garcia, R. B. Leal and M. B. Leal (2014). "Subchronic oral administration of Benzo[a]pyrene impairs motor and cognitive behavior and modulates S100B levels and MAPKs in rats." Neurochem Res 39(4): 731-740.

Madathil, S. K., P. T. Nelson, K. E. Saatman and B. R. Wilfred (2011). "MicroRNAs in CNS injury: potential roles and therapeutic implications." Bioessays 33(1): 21-26.

Mattick, J. S. and I. V. Makunin (2006). "Non-coding RNA." Hum Mol Genet 15 Spec No 1: R17-29.

McCallister, M. M., Z. Li, T. Zhang, A. Ramesh, R. S. Clark, M. Maguire, B. Hutsell, M. C. Newland and D. B. Hood (2015). "Revealing Behavioral Learning Deficit Phenotypes Subsequent to in utero Exposure to Benzo(a)pyrene." ToxSci 9(29): 1-43.

McCallister, M. M., M. Maguire, A. Ramesh, Q. Aimin, S. Liu, H. Khoshbouei, M. Aschner, F. F. Ebner 
and D. B. Hood (2008). "Prenatal exposure to benzo(a)pyrene impairs later-life cortical neuronal function." Neurotoxicology 29(5): 846-854.

Moore, L. D., T. Le and G. Fan (2013). "DNA methylation and its basic function." Neuropsychopharmacology 38(1): 23-38.

Morris, M. J. and L. M. Monteggia (2014). "Role of DNA methylation and the DNA methyltransferases in learning and memory." Dialogues Clin Neurosci 16(3): 359-371.

Nicholson, J. K. and J. C. Lindon (2008). "Systems biology: Metabonomics." Nature 455(7216): 1054-1056.

Pink, M., N. Verma, A. W. Rettenmeier and S. Schmitz-Spanke (2014). "Integrated proteomic and metabolomic analysis to assess the effects of pure and benzo[a]pyrene-loaded carbon black particles on energy metabolism and motility in the human endothelial cell line EA.hy926." Arch Toxicol 88(4): 913-934.

Ponjavic, J., P. L. Oliver, G. Lunter and C. P. Ponting (2009). "Genomic and transcriptional co-localization of protein-coding and long non-coding RNA pairs in the developing brain." PLoS Genet 5(8): e1000617.

Pooters, T., A. Van der Jeugd, Z. Callaerts-Vegh and R. D'Hooge (2015). "Telencephalic neurocircuitry and synaptic plasticity in rodent spatial learning and memory." Brain Res 1621: 294-308.

Popa-Wagner, A., S. Mitran, S. Sivanesan, E. Chang and A. M. Buga (2013). "ROS and brain diseases: the good, the bad, and the ugly." Oxid Med Cell Longev 2013: 963520.

Qiu, C., S. Cheng, Y. Xia, B. Peng, Q. Tang and B. Tu (2011). "Effects of subchronic benzo(a)pyrene exposure on neurotransmitter receptor gene expression in the rat hippocampus related with spatial learning and memory change." Toxicology 289(2-3): 83-90.

Qureshi, I. A., J. S. Mattick and M. F. Mehler (2010). "Long non-coding RNAs in nervous system function and disease." Brain Res 1338: 20-35.

Qureshi, I. A. and M. F. Mehler (2011). "Advances in epigenetics and epigenomics for neurodegenerative diseases." Curr Neurol Neurosci Rep 11(5): 464-473.

Ram'ırez, D. C., C. E. T. Vargas, S. G. Castillo, S. U. Carvajal, R. H. Pando, J. P. Chaverri and M. O. Ibarra (2012). "Effect of Glycolysis Inhibition on Mitochondrial Function in Rat Brain." J Biochem Molecular Toxicology 00(0): 1-6.

Rosinczuk, J., R. Dymarek and I. Calkosinski (2015). "Histopathological, ultrastructural, and immunohistochemical assessment of hippocampus structures of rats exposed to TCDD and high doses of tocopherol and acetylsalicylic acid." Biomed Res Int 2015: 645603.

Senut, M. C., A. Sen, P. Cingolani, A. Shaik, S. J. Land and D. M. Ruden (2014). "Lead exposure disrupts global DNA methylation in human embryonic stem cells and alters their neuronal differentiation." Toxicol Sci 139(1): 142-161.

Seyedabadi, M., G. Fakhfouri, V. Ramezani, S. E. Mehr and R. Rahimian (2014). "The role of cell adhesion molecules in synaptic plasticity and memory." Exp Brain Res 232(2014): 723-738.

Seyedabadi, M., G. Fakhfouri, V. Ramezani, S. E. Mehr and R. Rahimian (2014). "The role of serotonin in memory: interactions with neurotransmitters and downstream signaling." Exp Brain Res 232(3): 723-738.

Shen, K. and T. Meyer (1999). "Dynamic control of CaMKII translocation and localization in hippocampal neurons by NMDA receptor stimulation." Science 284(5411): 162-166.

Sheng, M. and C. C. Hoogenraad (2007). "The postsynaptic architecture of excitatory synapses: a more quantitative view." Annu Rev Biochem 76: 823-847. 
Shonesy, B. C., N. Jalan-Sakrikar, V. S. Cavener and R. J. Colbran (2014). "CaMKII: a molecular substrate for synaptic plasticity and memory." Prog Mol Biol Transl Sci 122: 61-87.

Siegel, G., G. Obernosterer, R. Fiore, M. Oehmen, S. Bicker, M. Christensen, S. Khudayberdiev, P. F. Leuschner, C. J. Busch, C. Kane, K. Hubel, F. Dekker, C. Hedberg, B. Rengarajan, C. Drepper, H. Waldmann, S. Kauppinen, M. E. Greenberg, A. Draguhn, M. Rehmsmeier, J. Martinez and G. M. Schratt (2009). "A functional screen implicates microRNA-138-dependent regulation of the depalmitoylation enzyme APT1 in dendritic spine morphogenesis." Nat Cell Biol 11(6): 705-716.

Slotkin, T. A. and F. J. Seidler (2009). "Benzo[a]pyrene impairs neurodifferentiation in PC12 cells." Brain Res Bull 80(1-2): 17-21.

Spear, L. P. (2007). "Assessment of Adolescent Neurotoxicity Rationale and Methodological Considerations." Neurotoxicol Teratol 29(1): 1-9.

Tabatabaie, L., L. W. Klomp, R. Berger and T. J. de Koning (2010). "L-serine synthesis in the central nervous system: a review on serine deficiency disorders." Mol Genet Metab 99(3): 256-262.

Tchekalarova, J., H. Kubova and P. Mares (2005). "Postnatal caffeine exposure: effects on motor skills and locomotor activity during ontogenesis." Behav Brain Res 160(1): 99-106.

Thalhammer, A. and L. A. Cingolani (2014). "Cell adhesion and homeostatic synaptic plasticity." Neuropharmacology 78: 23-30.

Velculescu, V. E., L. Zhang, W. Zhou, J. Vogelstein, M. A. Basrai, D. E. Bassett, Jr., P. Hieter, B. Vogelstein and K. W. Kinzler (1997). "Characterization of the yeast transcriptome." Cell 88(2): 243-251.

Verma, N., M. Pink, A. W. Rettenmeier and S. Schmitz-Spanke (2012). "Review on proteomic analyses of benzo[a]pyrene toxicity." Proteomics 12(11): 1731-1755.

Viberg, H., W. Mundy and P. Eriksson (2008). "Neonatal exposure to decabrominated diphenyl ether (PBDE 209) results in changes in BDNF, CaMKII and GAP-43, biochemical substrates of neuronal survival, growth, and synaptogenesis." Neurotoxicology 29(1): 152-159.

Wang, F., G. Niu, X. Chen and F. Cao (2011). "Molecular imaging of microRNAs." Eur J Nucl Med Mol Imaging 38(8): 1572-1579.

Wang, X., J. Zhang, Q. Huang, A. Alamdar, M. Tian, L. Liu and H. Shen (2015). "Serum metabolomics analysis reveals impaired lipid metabolism in rats after oral exposure to benzo(a)pyrene." Mol Biosyst 11(3): 753-759.

Welch, K. M., J. S. Meyer, E. Chabi, Y. Miyakawa, Y. Itoh, N. Ishihara and A. N. Chee (1975). "Tyrosine uptake and neurotransmitter synthesis in ischemic brain after administration of alpha and beta adrengeric blocking agents to man." Trans Am Neurol Assoc 100: 256-259.

Wen, C., M. Zhang, Y. Zhang, F. Sun, J. Ma, L. Hu, G. Lin and X. Wang (2016). "Brain metabolomics in rats after administration of ketamine." Biomed Chromatogr 30(1): 81-84.

Wilusz, J. E., H. Sunwoo and D. L. Spector (2009). "Long noncoding RNAs: functional surprises from the RNA world." Genes Dev 23(13): 1494-1504.

Xia, Y., S. Cheng, J. He, X. Liu, Y. Tang, H. Yuan, L. He, T. Lu, B. Tu and Y. Wang (2011). "Effects of subchronic exposure to benzo[a]pyrene $(\mathrm{B}[\mathrm{a}] \mathrm{P})$ on learning and memory, and neurotransmitters in male Sprague-Dawley rat." Neurotoxicology 32(2): 188-198.

Xiao, W., Y. Cao, H. Long, Z. Luo, S. Li, N. Deng, J. Wang, X. Lu, T. Wang, S. Ning, L. Wang and B. Xiao (2017). "Genome-Wide DNA Methylation Patterns Analysis of Noncoding RNAs in Temporal Lobe Epilepsy Patients." Mol Neurobiol.

Xu, M. Y., Y. J. Sun, P. Wang, H. Y. Xu, L. P. Chen, L. Zhu and Y. J. Wu (2015). "Metabolomics analysis 
and biomarker identification for brains of rats exposed subchronically to the mixtures of low-dose cadmium and chlorpyrifos." Chem Res Toxicol 28(6): 1216-1223.

Yang, K., C. Chen, S. Cheng, X. Cao and B. Tu (2017). "Effects of benzo(a)pyrene exposure on the ATPase activity and calcium concentration in the hippocampus of neonatal rats." Int J Occup Med Environ Health 30(2): 203-211.

Yu, N. K., S. H. Baek and B. K. Kaang (2011). "DNA methylation-mediated control of learning and memory." Mol Brain 4: 5.

Zaitsu, K., Y. Hayashi, K. Suzuki, H. Nakayama, N. Hattori, R. Takahara, M. Kusano, H. Tsuchihashi and A. Ishii (2015). "Metabolome disruption of the rat cerebrum induced by the acute toxic effects of the synthetic cannabinoid MAM-2201." Life Sci 137: 49-55.

Zhang, C., Y. Zhao, Y. Wang, H. Wu, X. Fang and H. Chen (2014). "Deep RNA sequencing reveals that microRNAs play a key role in lactation in rats." J Nutr 144(8): 1142-1149.

Zhang, L., X. Liu, L. You, D. Zhou, Q. Wang, F. Li, M. Cong, L. Li, J. Zhao, D. Liu, J. Yu and H. Wu (2011). "Benzo(a)pyrene-induced metabolic responses in Manila clam Ruditapes philippinarum by proton nuclear magnetic resonance ((1)H NMR) based metabolomics." Environ Toxicol Pharmacol 32(2): 218-225.

Zhang, T., W. Wang, J. Huang, X. Liu, H. Zhang and N. Zhang (2016). "Metabolomic investigation of regional brain tissue dysfunctions induced by global cerebral ischemia." BMC Neurosci 17(1): 25.

Zhang, W., F. Tian, J. Zheng, S. Li and M. Qiang (2016). "Chronic Administration of Benzo(a)pyrene Induces Memory Impairment and Anxiety-Like Behavior and Increases of NR2B DNA Methylation." PLoS One 11(2): e0149574.

Zhang, X., L. Xu, D. He and S. Ling (2013). "Endoplasmic reticulum stress-mediated hippocampal neuron apoptosis involved in diabetic cognitive impairment." Biomed Res Int 2013: 924327. 
Table 1 Variation of weights of the rats throughout the study

\begin{tabular}{|c|c|c|c|c|c|c|c|c|}
\hline \multirow{2}{*}{ Group } & \multicolumn{8}{|c|}{ Week } \\
\hline & 0 & 1 & 2 & 3 & 4 & 5 & 6 & 7 \\
\hline \multicolumn{9}{|l|}{ Control group } \\
\hline weights (g) & $8.58 \pm 0.93$ & $14.88 \pm 3.23$ & $32.80 \pm 10.53$ & $49.68 \pm 12.88$ & $74.75 \pm 14.39$ & $101.40 \pm 17.29$ & $121.03 \pm 13.67$ & $142.48 \pm 21.41$ \\
\hline \multicolumn{9}{|l|}{$\mathrm{B}[\mathrm{a}] \mathrm{P}$ group } \\
\hline weights (g) & $7.68 \pm 1.98$ & $16.19 \pm 1.93$ & $25.36 \pm 2.75 *$ & $41.62 \pm 5.46 *$ & $64.65 \pm 9.27 *$ & $81.32 \pm 15.35$ * & $96.28 \pm 12.23 *$ & $118.13 \pm 11.01 *$ \\
\hline
\end{tabular}

$* P<0.05$, when compared with the control group.

Downloaded from https://academic.oup.com/toxsci/advance-article-abstract/doi/10.1093/toxsci/kfy192/5062621 by Leicester University Library user 
Table 2 KEGG and PANTHER pathways that related to learning and memory enriched for DEG

\begin{tabular}{|c|c|c|c|c|}
\hline Term & Database & ID & $P$-Value & No. of Genes \\
\hline Cell adhesion molecules (CAMs) & KEGG PATHWAY & rno04514 & $7.36277 \mathrm{E}-10$ & 25 \\
\hline Neuroactive ligand-receptor interaction & KEGG PATHWAY & rno04080 & $1.91779 \mathrm{E}-06$ & 26 \\
\hline Cytokine-cytokine receptor interaction & KEGG PATHWAY & rno04060 & $6.91005 \mathrm{E}-05$ & 19 \\
\hline ECM-receptor interaction & KEGG PATHWAY & rno04512 & 0.001 & 9 \\
\hline Tyrosine metabolism & KEGG PATHWAY & rno00350 & 0.002 & 6 \\
\hline Tryptophan metabolism & KEGG PATHWAY & rno00380 & 0.004 & 6 \\
\hline Retinol metabolism & KEGG PATHWAY & rno00830 & 0.005 & 8 \\
\hline PI3K-Akt signaling pathway & KEGG PATHWAY & rno04151 & 0.007 & 19 \\
\hline Linoleic acid metabolism & KEGG PATHWAY & rno00591 & 0.010 & 5 \\
\hline Jak-STAT signaling pathway & KEGG PATHWAY & rno04630 & 0.018 & 10 \\
\hline TNF signaling pathway & KEGG PATHWAY & rno04668 & 0.020 & 8 \\
\hline Chemokine signaling pathway & KEGG PATHWAY & rno04062 & 0.021 & 11 \\
\hline Hippo signaling pathway & KEGG PATHWAY & rno04390 & 0.022 & 10 \\
\hline GnRH signaling pathway & KEGG PATHWAY & rno04912 & 0.024 & 7 \\
\hline Toll-like receptor signaling pathway & KEGG PATHWAY & rno04620 & 0.030 & 7 \\
\hline NOD-like receptor signaling pathway & KEGG PATHWAY & rno04621 & 0.034 & 5 \\
\hline Arachidonic acid metabolism & KEGG PATHWAY & rno00590 & 0.039 & 6 \\
\hline Vitamin B6 metabolism & KEGG PATHWAY & rno00750 & 0.045 & 2 \\
\hline Alzheimer disease-presenilin pathway & PANTHER & P00004 & 0.016 & 7 \\
\hline Cadherin signaling pathway & PANTHER & P00012 & 0.024 & 6 \\
\hline TGF-beta signaling pathway & PANTHER & $\mathrm{P} 00052$ & 0.034 & 5 \\
\hline
\end{tabular}

* The DEG involved in the pathways are shown in supplementary table 1. 
Table 3 KEGG and PANTHER pathways that related to learning and memory enriched for differentially expressed methylated ncRNAs predicted target genes

\begin{tabular}{|c|c|c|c|c|}
\hline Term & Database & ID & $P$-Value & No. of Genes \\
\hline MAPK signaling pathway & KEGG PATHWAY & rno04010 & $9.3126 \mathrm{E}-06$ & 9 \\
\hline Hippo signaling pathway & KEGG PATHWAY & rno04390 & 0.001 & 5 \\
\hline Rap1 signaling pathway & KEGG PATHWAY & rno04015 & 0.005 & 5 \\
\hline Phospholipase D signaling pathway & KEGG PATHWAY & rno04072 & 0.007 & 4 \\
\hline Cell adhesion molecules (CAMs) & KEGG PATHWAY & rno04514 & 0.013 & 4 \\
\hline Apoptosis - multiple species & KEGG PATHWAY & rno04215 & 0.013 & 2 \\
\hline TNF signaling pathway & KEGG PATHWAY & rno04668 & 0.020 & 3 \\
\hline Neurotrophin signaling pathway & KEGG PATHWAY & rno04722 & 0.028 & 3 \\
\hline Glutathione metabolism & KEGG PATHWAY & rno00480 & 0.038 & 2 \\
\hline Long-term potentiation & KEGG PATHWAY & rno04720 & 0.045 & 2 \\
\hline Wnt signaling pathway & PANTHER & P00057 & 0.000 & 6 \\
\hline EGF receptor signaling pathway & PANTHER & P00018 & 0.001 & 4 \\
\hline Glycolysis & PANTHER & P00024 & 0.003 & 2 \\
\hline FGF signaling pathway & PANTHER & P00021 & 0.008 & 3 \\
\hline Beta2 adrenergic receptor signaling pathway & PANTHER & P04378 & 0.009 & 2 \\
\hline Beta1 adrenergic receptor signaling pathway & PANTHER & P04377 & 0.010 & 2 \\
\hline Toll receptor signaling pathway & PANTHER & P00054 & 0.011 & 2 \\
\hline Integrin signalling pathway & PANTHER & P00034 & 0.017 & 3 \\
\hline Oxytocin receptor mediated signaling pathway & PANTHER & P04391 & 0.018 & 2 \\
\hline VEGF signaling pathway & PANTHER & P00056 & 0.022 & 2 \\
\hline $5 \mathrm{HT} 2$ type receptor mediated signaling pathway & PANTHER & P04374 & 0.026 & 2 \\
\hline p53 pathway & PANTHER & P00059 & 0.027 & 2 \\
\hline Parkinson disease & PANTHER & P00049 & 0.044 & 2 \\
\hline
\end{tabular}

* The differentially expressed methylated ncRNAs predicted target genes involved in the pathways are shown in supplementary table 2 . 
Table 4 Metabolites significantly changed in hippocampus

\begin{tabular}{|c|c|c|c|c|c|}
\hline Name & $P$-Value & VIP* & $\mathrm{RT}(\min )^{*}$ & Match (\%) & $\mathrm{FC}^{*}$ \\
\hline N-Acetylglutamic acid & 0.0493321 & 1.20956 & 19.507 & 74 & 1.0231955 \\
\hline Glycine & 0.0477313 & 1.24744 & 11.665 & 96 & 1.0211368 \\
\hline Glyoxylic acid & 0.0330821 & 1.25821 & 11.414 & 74 & 1.074236 \\
\hline Isoleucine & 0.0114773 & 1.35729 & 14.297 & 94 & 1.0359669 \\
\hline Leucine & 0.0085528 & 1.38005 & 14.242 & 98 & 1.0353758 \\
\hline Benzoic acid & 0.0324072 & 1.38424 & 9.861 & 72 & -1.112152 \\
\hline 2-Hydroxyisobutyric acid & 0.0399133 & 1.40399 & 9.359 & 74 & 1.0588779 \\
\hline Hexanoic acid & 0.008316 & 1.407 & 6.514 & 82 & 1.0524409 \\
\hline Phenylalanine & 0.0068899 & 1.41187 & 20.305 & 96 & 1.0252458 \\
\hline Proline & 0.0045741 & 1.4237 & 15.242 & 98 & 1.0212806 \\
\hline Aspartic acid & 0.0246484 & 1.43881 & 16.578 & 98 & 1.0061667 \\
\hline Phosphoenolpyruvic acid & 0.0166334 & 1.48326 & 14.765 & 63 & -1.17109874 \\
\hline Valine & 0.0009839 & 1.50823 & 12.992 & 92 & 1.0275114 \\
\hline 4-Hydroxyphenylpyruvate & 0.0257091 & 1.51697 & 16.066 & 70 & -1.031166 \\
\hline Dehydroascorbic acid & 0.0011967 & 1.53076 & 15.784 & 86 & 1.0298389 \\
\hline Cysteine & 0.0400343 & 1.62554 & 20.466 & 90 & -1.056389 \\
\hline Threonine & 0.0132601 & 1.73652 & 15.857 & 97 & 1.0175589 \\
\hline Caprylic acid & 0.0111353 & 1.79551 & 9.694 & 88 & -1.017844 \\
\hline Histidine & 0.0081575 & 1.38474 & 27.545 & 88 & -1.03299 \\
\hline Fumaric acid & 0.0314558 & 1.45179 & 9.419 & 84 & -1.024587 \\
\hline cis-Aconitic acid & 0.0472475 & 1.53995 & 15.711 & 90 & -1.017213 \\
\hline
\end{tabular}

*Fold change $(\mathrm{FC})$ between $\mathrm{B}[\mathrm{a}] \mathrm{P}$-treated rats and control rats. Fold change with a positive value indicates a higher concentration in $\mathrm{B}[\mathrm{a}] \mathrm{P}$-treated rats, while a negative value means a relatively lower concentration as compared to the control rats.

*RT retention time.

*VIP variable importance in the projection obtained from PLS-DA model with a threshold of 1.0. 
Table 5 Metabolic pathways in the hippocampus which were altered by B[a]P exposure

\begin{tabular}{|c|c|c|c|c|c|c|c|}
\hline Name & Total* & Hit* & $P$ value* & $-\log (P)$ & Holm $P$ & FDR & Impact* \\
\hline \multicolumn{8}{|l|}{ Ubiquinone and other terpenoid-quinone } \\
\hline & 3 & 1 & 0.042218 & 3.1649 & 1 & 0.27561 & 1 \\
\hline \multicolumn{8}{|l|}{ biosynthesis } \\
\hline Valine, leucine and isoleucine biosynthesis & 11 & 3 & 0.00038144 & 7.8716 & 0.030515 & 0.015448 & 0.99999 \\
\hline \multicolumn{8}{|l|}{ Phenylalanine, tyrosine and tryptophan } \\
\hline & 4 & 2 & 0.001141 & 6.7759 & 0.088996 & 0.023105 & 0.5 \\
\hline \multicolumn{8}{|l|}{ biosynthesis } \\
\hline Phenylalanine metabolism & 9 & 1 & 0.12163 & 2.1068 & 1 & 0.4926 & 0.40741 \\
\hline Glycine, serine and threonine metabolism & 32 & 4 & 0.00083954 & 7.0827 & 0.066323 & 0.022667 & 0.29197 \\
\hline Histidine metabolism & 15 & 2 & 0.01817 & 4.008 & 1 & 0.14718 & 0.24194 \\
\hline Alanine, aspartate and glutamate metabolism & 24 & 2 & 0.044234 & 3.1183 & 1 & 0.27561 & 0.1962 \\
\hline Glyoxylate and dicarboxylate metabolism & 16 & 2 & 0.020589 & 3.883 & 1 & 0.15161 & 0.14815 \\
\hline Cysteine and methionine metabolism & 28 & 1 & 0.33386 & 1.097 & 1 & 1 & 0.12829 \\
\hline Glycolysis or Gluconeogenesis & 26 & 1 & 0.31406 & 1.1582 & 1 & 1 & 0.10389 \\
\hline Tyrosine metabolism & 42 & 2 & 0.11853 & 2.1326 & 1 & 0.4926 & 0.07225 \\
\hline Citrate cycle (TCA cycle) & 20 & 3 & 0.0024271 & 6.0211 & 0.18688 & 0.038287 & 0.06496 \\
\hline Arginine and proline metabolism & 44 & 4 & 0.0028361 & 5.8653 & 0.21554 & 0.038287 & 0.064 \\
\hline Primary bile acid biosynthesis & 46 & 1 & 0.48924 & 0.7149 & 1 & 1 & 0.02976 \\
\hline Glutathione metabolism & 26 & 2 & 0.05121 & 2.9718 & 1 & 0.29629 & 0.00955 \\
\hline
\end{tabular}

*Total is the total number compounds in the pathway;

*Hit is the matched number from our uploaded metabolites;

* $P$ value and FDR calculated from the enrichment analysis;

*Impact value calculated from the pathway topology analysis. 
Table 6 Differential genes that can regulate the expression of significant metabolites by regulating enzymes

\begin{tabular}{ccccccccc}
\hline Gene & $P$-value & Fold change & Gene & $P$-value & Fold change & Gene & $P$-value & Fold change \\
\hline Eno1 & 0.010 & 1.047 & Aga & 0.009 & -1.129 & Ass1 & 0.002 & 1.267 \\
Adss & 0.011 & 1.060 & Got1 & $1.57 \mathrm{E}-23$ & 1.135 & Sardh & $1.46 \mathrm{E}-08$ & 1.276 \\
Bcat1 & $1.54 \mathrm{E}-34$ & 1.079 & Cbs & $6.24 \mathrm{E}-11$ & 1.145 & Adss1 & 0.050 & 1.345 \\
Sdhb & $3.16 \mathrm{E}-05$ & 1.083 & Nans & $2.00 \mathrm{E}-08$ & 1.153 & Cpa2 & 0.007 & -1.567 \\
Sdhc & $2.73 \mathrm{E}-07$ & 1.086 & Asns & $5.60 \mathrm{E}-11$ & 1.162 & Pipox & 0.015 & 1.722 \\
Got2 & $7.45 \mathrm{E}-20$ & 1.090 & Eno1 & $2.94 \mathrm{E}-91$ & 1.166 & Tpo & 0.029 & -3.775 \\
Fh & 0.001 & 1.092 & Gatm & $2.80 \mathrm{E}-15$ & 1.167 & Dpep2 & 0.000 & -8.966 \\
Sdha & $7.82 \mathrm{E}-24$ & 1.109 & Ads1 & $4.58 \mathrm{E}-13$ & 1.177 & & & \\
Pck2 & 0.000 & 1.129 & Gad1 & $1.98 \mathrm{E}-39$ & 1.195 & & & \\
\hline
\end{tabular}

*Fold change between $\mathrm{B}[\mathrm{a}] \mathrm{P}$-treated rats and control rats. Fold change with a positive value indicates a higher concentration, while a negative value indicates a relatively lower concentration present in $\mathrm{B}[\mathrm{a}] \mathrm{P}-$ treated rats as compared to the control rats.

Downloaded from https://academic.oup.com/toxsci/advance-article-abstract/doi/10.1093/toxsci/kfy192/5062621 by Leicester University Library user 


\section{Figure Legends}

Fig. 1 Performance of the Morris Water Maze: (A) Swim speed, data was expressed as Mean $\pm S D(n=8)$ of average swim speed made on different days $(F=0.520, P=0.483)$; (B) Escape latency, in seconds, to reach the platform during each trial. Data was expressed as Mean $\pm S D(n=8)$ of average escape latency made on different days $(F=6.555, P=0.023)$; (C) The number of platform crossings, data sows the average frequency of platform crossings made on day 5. The height of each bar represents the Mean $\pm S D(n=5)(F=6.216, P=0.000)$; (D) Time spent in target area, data shows the average time spent in the target quadrant on day 5 . The height of each bar represents the Mean $\pm S D(n=5)(F=3.494, P=0.014)$; (E) Representative photographs of rats' swimming tracks. * $P<0.05$, compared with the control group.

Fig. 2 Representative electron micrographs of sections of rat hippocampal neurons. (A) Control group; (B) $\mathrm{B}[\mathrm{a}] \mathrm{P}$-treated group; (C) PSD thickness, data was expressed as Mean $\pm S D(n=50)$; (D) Normal myelinated fibers, with plasma membrane rings of regular heights and dense structure; (E) Damaged myelin sheaths, with stratification and collapse; (F) Normal mitochondria; (G) Swelled mitochondria. The arrows show the synaptic linkages. $* P<0.05$, compared with the control group. Magnification $\times 20,000$.

Fig. 3 Expression profiles of miRNAs, mRNAs, and lncRNAs. (A) Volcano plots of all detected miRNAs in both the control group and B[a]P-treated group. The vertical dotted line delimits up-regulation and down-regulation. Red plots represent significant mRNAs with fold change $>2.0, P<0.05$; (B) Volcano plots of all detected mRNAs in both control group and B[a]P-treated group; (C) Volcano plots of all detected lncRNAs in both control group and $\mathrm{B}[\mathrm{a}] \mathrm{P}$-treated group.

Fig. 4 B[a]P induced DNA methylation. (A) DNA methylation of the CGI and promoter regions. (B) DNA methylation signal genomic element annotation. (C) Distribution of DMRs and DMCs on the chromosome. The inner ring and the outer ring exhibits DMRs and DMCs, respectively.

Fig. 5 Partial least squares discriminate analysis (PLS-DA) score plot representing the metabolomic profiles of the hippocampus in the $\mathrm{B}[\mathrm{a}] \mathrm{P}$-treated and control groups $(\mathrm{R} 2 \mathrm{X}=0.743, \mathrm{R} 2 \mathrm{Y}=0.993, \mathrm{Q} 2=0.596)$. 
Fig. 6 Heat map of metabolites that were significantly different between the control group and B[a]P-treated group. Red indicates upregulation, green indicates downregulation.

Fig. 7 All match pathway $P$ values from pathway enrichment analysis and pathway impact value from pathway topology analysis by Metaboanalyst. Enriched metabolic pathways were ranked by false discovery rate (FDR) values. Significant pathways were represented by bigger/red dots and $\log P$ value. Pathway impact was calculated as the sum of the importance measures of the matched metabolites normalized by the sum of the importance measures of all metabolites in each pathway. B[a]P altered metabolic pathways of the hippocampus were shown in Table 5.

Fig. 8 Enzyme gene compounds reaction network of the significant metabolites. Figures in red represent significant metabolites, those in pink represent intermediate metabolites; green squares represent enzymes, and violet circles represent genes.

Fig. 9 A model illustrating the possible regulated processes intertwined by DNA methylation, ncRNAs, and coding genes, after exposure to B[a]P. IncRNAs and miRNAs cooperatively mediate the pathway dysregulation which may be implicated in $\mathrm{B}[\mathrm{a}] \mathrm{P}$-induced learning and memory dysfunction.

Downloaded from https://academic.oup.com/toxsci/advance-article-abstract/doi/10.1093/toxsci/kfy192/5062621 by Lelcester 
A
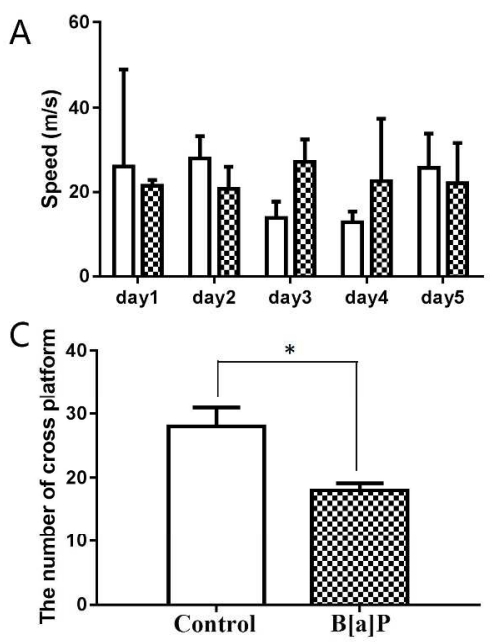

$\mathrm{E}$

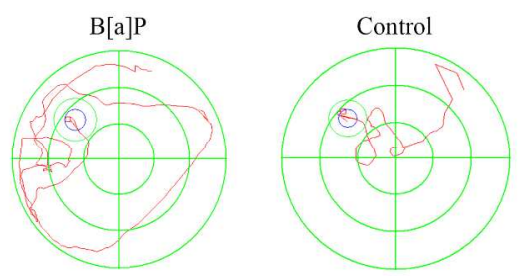

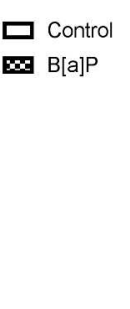

口 Control $\mathrm{B} \mathrm{B}[\mathrm{a}] \mathrm{P}$

D
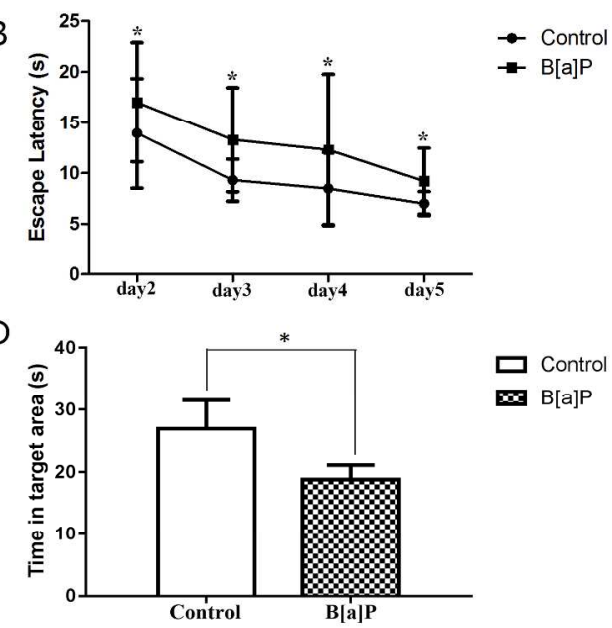

Fig. 1 Performance of the Morris Water Maze: (A) Swim speed, data was expressed as Mean \pm SD $(n=8)$ of average swim speed made on different days $(F=0.520, P=0.483)$; $(B)$ Escape latency, in seconds, to reach the platform during each trial. Data was expressed as Mean $\pm S D(n=8)$ of average escape latency made on different days $(F=6.555, P=0.023)$; (C) The number of platform crossings, data sows the average frequency of platform crossings made on day 5 . The height of each bar represents the Mean \pm SD $(n=5)(F=6.216, P=0.000)$; (D) Time spent in target area, data shows the average time spent in the target quadrant on day 5. The height of each bar represents the Mean $\pm S D(n=5)(F=3.494, P=0.014)$; (E) Representative photographs of rats' swimming tracks. * $\mathrm{P}<0.05$, compared with the control group.

$259 \times 191 \mathrm{~mm}(300 \times 300 \mathrm{DPI})$ 

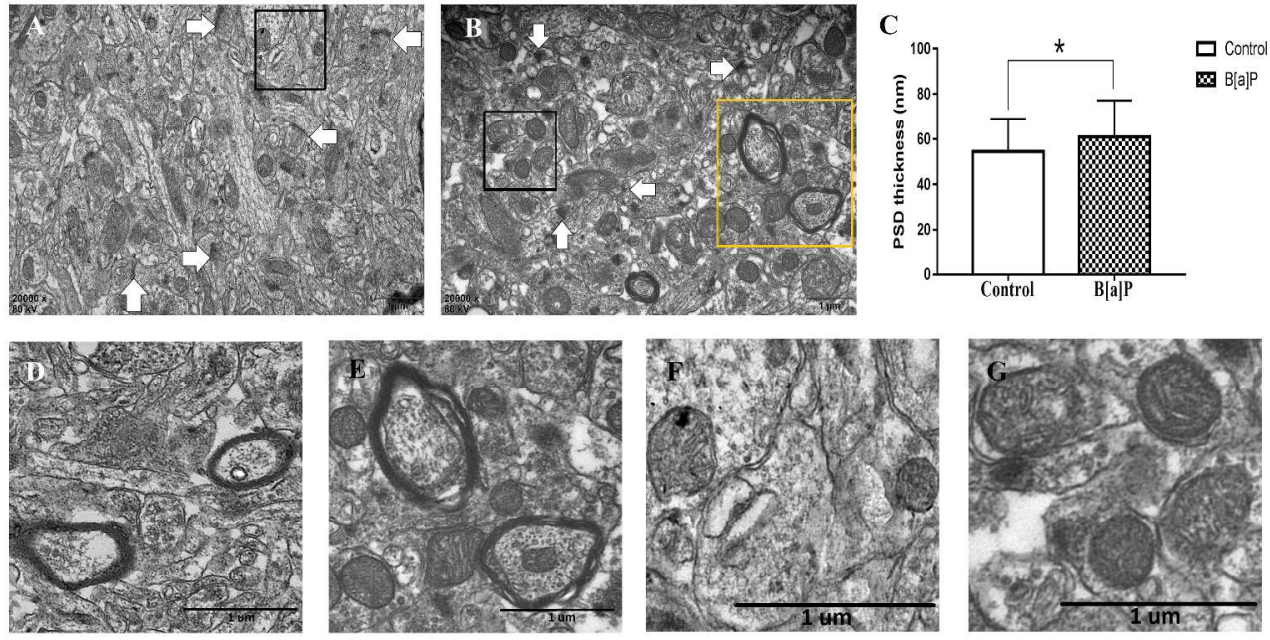

Fig. 2 Representative electron micrographs of sections of rat hippocampal neurons. (A) Control group; (B) $B$ [a]P-treated group; (C) PSD thickness, data was expressed as Mean \pm SD $(n=50)$; (D) Normal myelinated fibers, with plasma membrane rings of regular heights and dense structure; (E) Damaged myelin sheaths, with stratification and collapse; (F) Normal mitochondria; (G) Swelled mitochondria. The arrows show the synaptic linkages. $* P<0.05$, compared with the control group. Magnification $\times 20,000$.

$399 \times 205 \mathrm{~mm}(300 \times 300$ DPI $)$ 

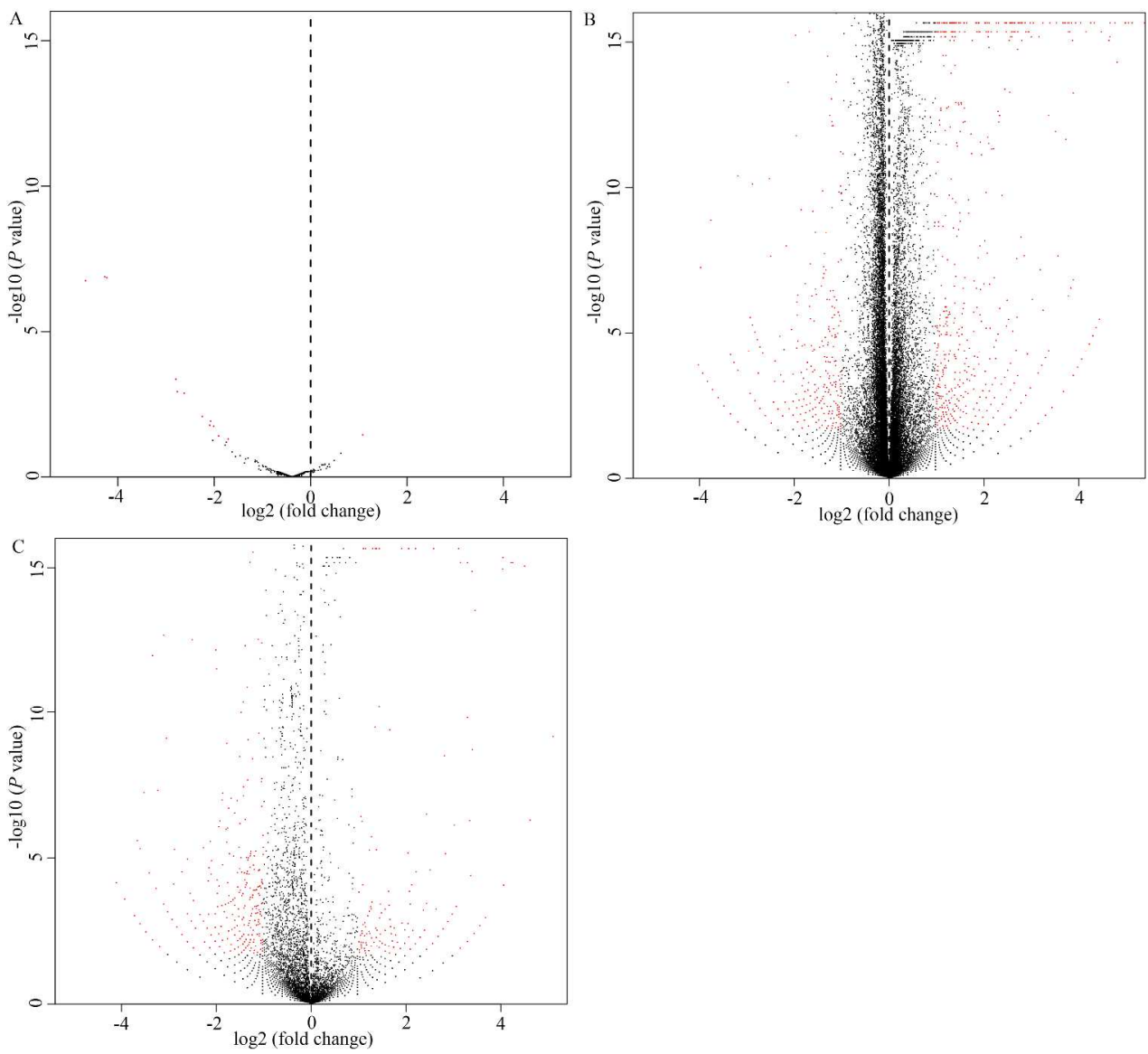

Fig. 3 Expression profiles of miRNAs, mRNAs, and IncRNAs. (A) Volcano plots of all detected miRNAs in both the control group and B[a]P-treated group. The vertical dotted line delimits up-regulation and downregulation. Red plots represent significant mRNAs with fold change $>2.0, \mathrm{P}<0.05 ;(\mathrm{B})$ Volcano plots of all detected mRNAs in both control group and B[a]P-treated group; (C) Volcano plots of all detected IncRNAs in both control group and $\mathrm{B}[\mathrm{a}] \mathrm{P}$-treated group.

$394 \times 358 \mathrm{~mm}(300 \times 300$ DPI) 


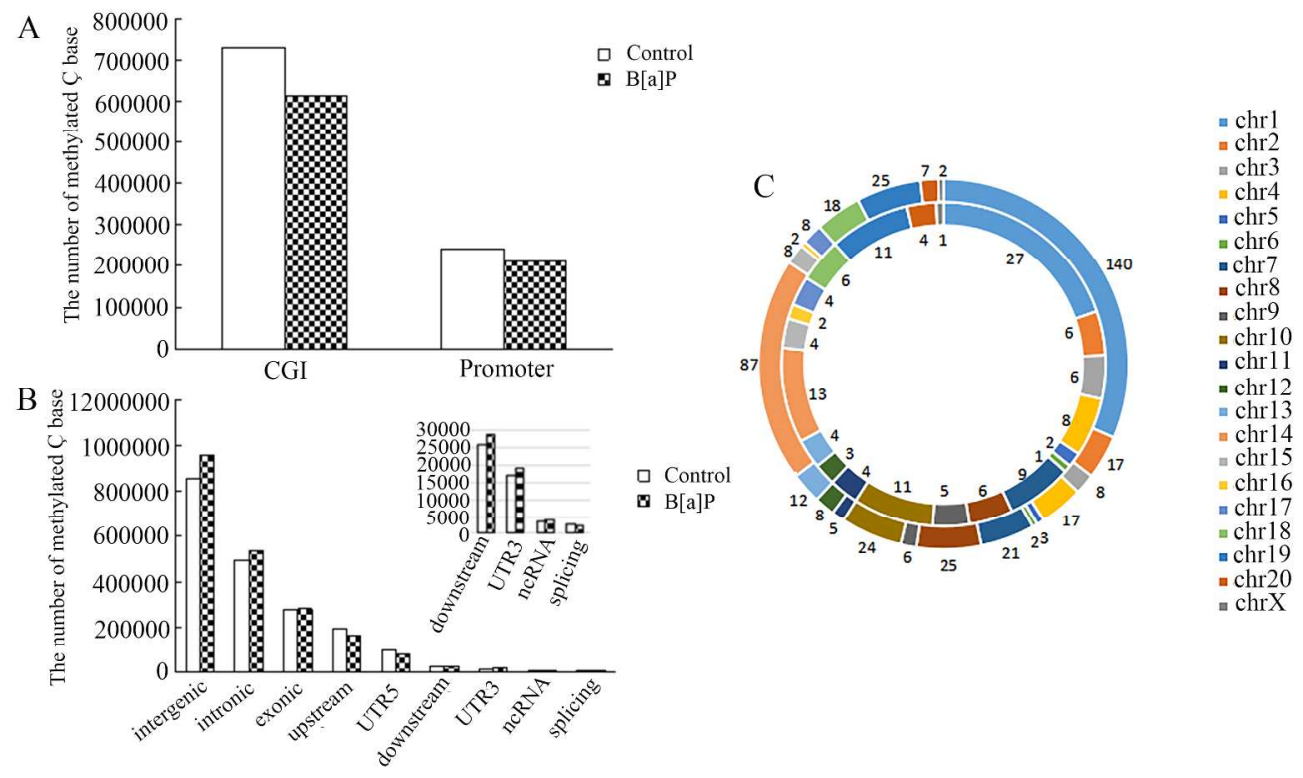

Fig. $4 \mathrm{~B}[\mathrm{a}] \mathrm{P}$ induced DNA methylation. (A) DNA methylation of the CGI and promoter regions. (B) DNA methylation signal genomic element annotation. (C) Distribution of DMRs and DMCs on the chromosome. The inner ring and the outer ring exhibits DMRs and DMCs, respectively.

$251 \times 149 \mathrm{~mm}(300 \times 300$ DPI) 


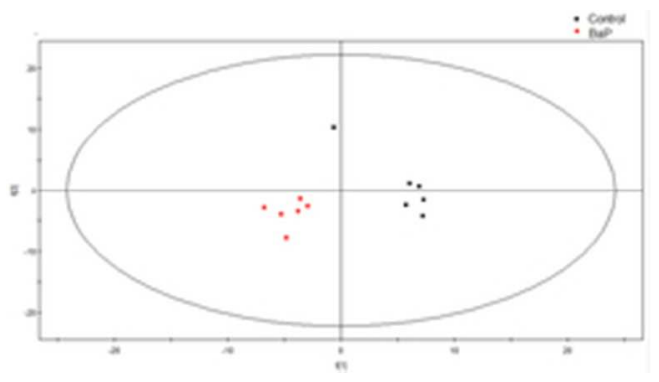

Fig. 5 Partial least squares discriminate analysis (PLS-DA) score plot representing the metabolomic profiles of the hippocampus in the $\mathrm{B}[\mathrm{a}] \mathrm{P}$-treated and control groups $(\mathrm{R} 2 \mathrm{X}=0.743, \mathrm{R} 2 \mathrm{Y}=0.993, \mathrm{Q} 2=0.596)$.

$20 \times 11 \mathrm{~mm}(300 \times 300 \mathrm{DPI})$

Downloaded from https://academic.oup.com/toxsci/advance-article-abstract/doi/10.1093/toxsci/kfy192/5062621 by Leicester University Library user 


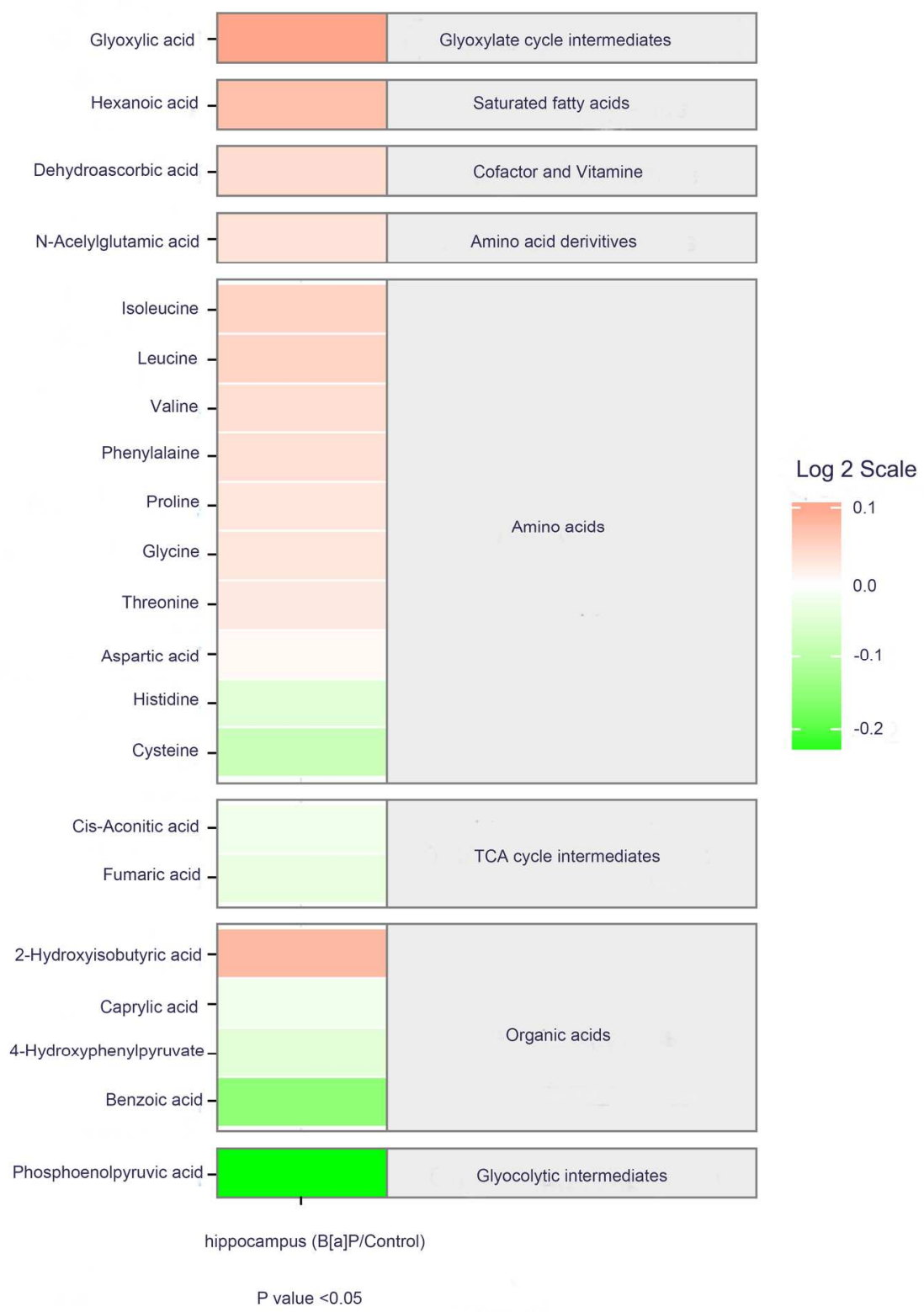

Fig. 6 Heat map of metabolites that were significantly different between the control group and B[a]P-treated group. Red indicates upregulation, green indicates downregulation.

$161 \times 228 \mathrm{~mm}(300 \times 300 \mathrm{DPI})$ 


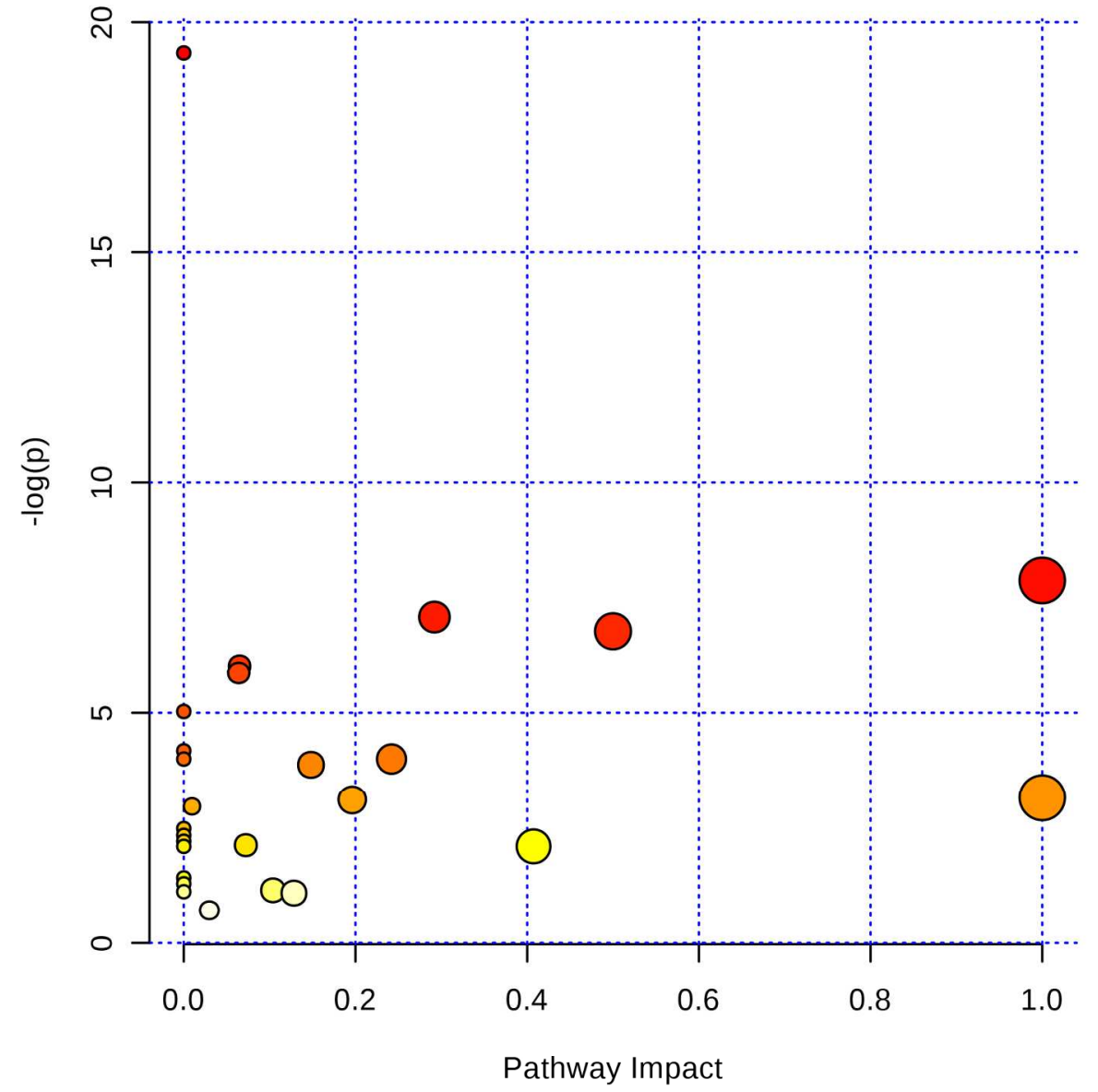

Fig. 7 All match pathway $P$ values from pathway enrichment analysis and pathway impact value from pathway topology analysis by Metaboanalyst. Enriched metabolic pathways were ranked by false discovery rate (FDR) values. Significant pathways were represented by bigger/red dots and Log P value. Pathway impact was calculated as the sum of the importance measures of the matched metabolites normalized by the sum of the importance measures of all metabolites in each pathway. B[a]P altered metabolic pathways of the hippocampus were shown in Table 5.

$177 \times 177 \mathrm{~mm}(300 \times 300 \mathrm{DPI})$ 


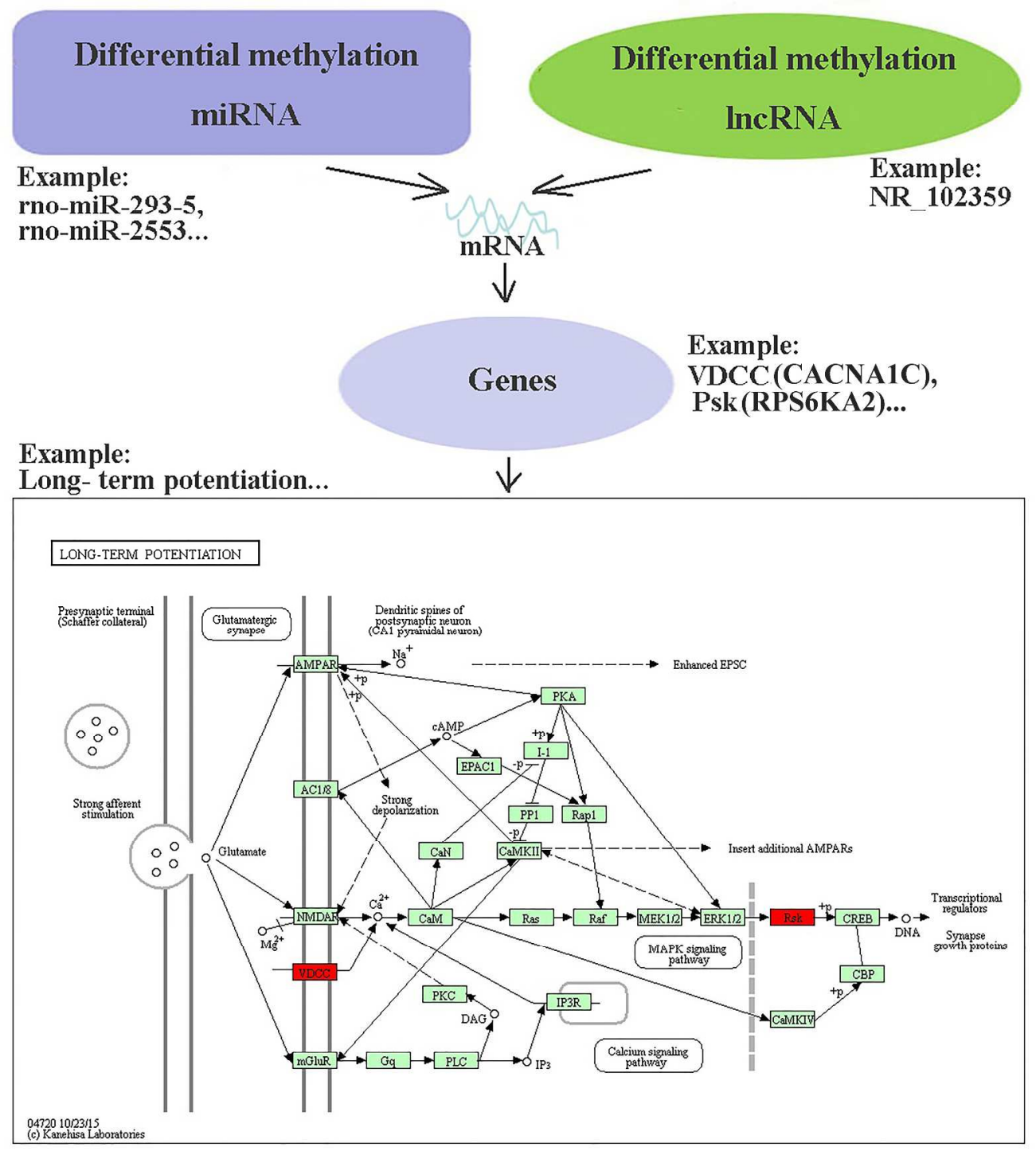

Fig. 9 A model illustrating the possible regulated processes intertwined by DNA methylation, ncRNAs, and coding genes, after exposure to B[a]P. IncRNAs and miRNAs cooperatively mediate the pathway dysregulation which may be implicated in $\mathrm{B}[\mathrm{a}] \mathrm{P}$-induced learning and memory dysfunction.

$$
383 \times 427 \mathrm{~mm}(300 \times 300 \mathrm{DPI})
$$

